\title{
IL-12 from endogenous CDC1, and not vaccine $D C$, is required for Th1 induction
}

\author{
DiyaaEIDin Ashour, ${ }^{1}$ Panagiota Arampatzi, ${ }^{2}$ Vladimir Pavlovic, ${ }^{1}$ Konrad U. Förstner, ${ }^{2,3,4}$ \\ Tsuneyasu Kaisho, ${ }^{5}$ Andreas Beilhack, ${ }^{6}$ Florian Erhard, ${ }^{1}$ and Manfred B. Lutz ${ }^{1}$ \\ ${ }^{1}$ Institute for Virology and Immunobiology, and ${ }^{2}$ Core Unit Systems Medicine, University of Würzburg, Würzburg, \\ Germany. ${ }^{3} \mathrm{ZB}$ MED, Information Centre for Life Sciences, Cologne, Germany. ${ }^{4} \mathrm{TH}$ Köln, University of Applied Sciences, \\ Institute of Information Science, Cologne, Germany. ${ }^{5}$ Department of Immunology, Institute of Advanced Medicine, \\ Wakayama Medical University, Wakayama, Wakayama, Japan. ${ }^{6}$ Department of Internal Medicine II, University Hospital \\ Würzburg, Würzburg, Germany.
}

Success of DC vaccines relies on the quality of antigen presentation, costimulation, lymph node migration, and the release of IL-12, in case of Th1 priming. Here, we provide evidence for interaction between the injected vaccine DCs with endogenous lymph node-resident DCs for Th1 induction. While migration of the injected DCs was essential for antigen delivery to the lymph node, the injected DCs contributed only partially to ThO priming and were unable to instruct Th1 generation. Instead, we provide evidence that the lymph node-resident XCR1+ DCs are activated by the injected DCs to present the cognate antigen and release IL-12 for Th1 polarization. The timing of interactions in the draining lymph nodes appeared step-wise as (a) injected DCs with cognate T cells, (b) injected DCs with bystander DCs, and (c) bystander DCs with T cells. The transcriptome of the bystander DCs showed a downregulation of Treg- and Th2/Th9-inducing genes and self-antigen presentation, as well as upregulation of MHC class II and genes required for Th1 instruction. Together, these data show that injected mature lymph node migratory DCs direct T cell priming and bystander DC activation, but not Th1 polarization, which is mediated by endogenous IL-12p70+XCR1 ${ }^{+}$resident bystander DCs. Our results are of importance for clinical DC-based vaccinations against tumors where endogenous DCs may be functionally impaired by chemotherapy.

Conflict of interest: The authors have declared that no conflict of interest exists.

Copyright: ( 2020 , Ashour et al. This is an open access article published under the terms of the Creative Commons Attribution 4.0 International License.

Submitted: November 20, 2019

Accepted: April 22, 2020

Published: May 21, 2020.

Reference information: /CI Insight. 2020;5(10):e135143.

https://doi.org/10.1172/jci. insight.135143.

\section{Introduction}

DCs play a central role in priming and polarization of naive CD4 ${ }^{+} \mathrm{T}$ cells into subsequent Th subsets (1). DCs are composed of defined subsets with different ontogenies, surface markers, functions, and spatial organization within lymphoid organs (2-4). Plasmacytoid DCs (pDCs) and 2 subsets of conventional DCs (cDCs) can act tolerogenic (5-8) but also as sentinels for pathogen recognition for subsequent $\mathrm{CD}^{+} \mathrm{T}$ cell priming and polarization toward Th1, Th2, or Th17 helper responses, depending on the subset and the pathogen-directed signals $(9,10)$

pDCs are a major source of IFN- $\alpha$ for antiviral responses (11), while cDCs are classified into XCR $1^{+}$

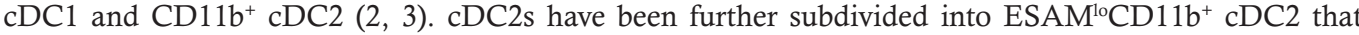
are dependent on Klf4 transcription and functionally induce Th2 responses (12), while $\mathrm{ESAM}^{\mathrm{hi}} \mathrm{CD} 11 \mathrm{~b}^{+}$ cDC2 are dependent on the Notch2 transcription factor and preferentially induce Th17 responses (13) The $\mathrm{CD}^{+}$or $\mathrm{CD} 103^{+} \mathrm{XCR}^{+} \mathrm{cDC} 1$ subset is specialized in MHC class I-dependent (MHC-I-dependent) cross-presentation for $\mathrm{CD}^{+} \mathrm{T}$ cell responses (14), as well as in $\mathrm{CD}^{+} \mathrm{Th} 1$ polarization against intracellular pathogens and tumors (10).

Under inflammatory conditions, another subset of DCs of monocytic origin (MoDC) is generated that are characterized by expression of macrophage-related markers $\mathrm{CD} 64^{+} \mathrm{CD} 11 \mathrm{~b}^{+} \mathrm{MAR} 1^{+}(15)$. MoDCs represent a reservoir for rapid recruitment of DCs during inflammation (16). In vitro GM-CSF generated BM-derived DCs (BM-DCs) resemble MoDCs (17) and such BM-DCs can be instructed by pathogens or inflammatory signals to induce Th1, Th2, and Th17 responses, depending on the quality and magnitude of the stimulation $(18,19)$.

The induction of Th1 responses by DCs relies on 3 distinct stimuli from DCs. MHC peptide complexes ligating the TCR (signal 1) and CD80/CD86 costimulation (signal 2) lead to T cell activation and proliferation, reaching a stage termed Th0, where IL-2 production by the T cells can be measured, but 
without secretion of Th1 cytokines such as IFN- $\gamma$ (20). Signal 3 has been proposed for the induction of polarized CD4 ${ }^{+}$Th responses (21), and the heterodimeric IL-12p70 cytokine turned out to be critical for the induction of Th1 cells (22). IL-12 production by DCs can be induced by different pathogen signals but not proinflammatory cytokines (23).

Human MoDCs can be derived from CD14+ peripheral blood monocytes (24) and are the prime candidates for adoptive DC vaccine trials in tumor patients (25). Still, the most common DC vaccination approach is based on MoDCs $(26,27)$, and they are typically matured by a cytokine cocktail consisting of IL-1 $\beta / \mathrm{TNF}-\alpha / \mathrm{IL}-6 / \mathrm{PGE}_{2}$ (28). Although tumor therapy with cocktail-matured MoDCs has proven to be successful in melanoma patients, it is still unclear how such cytokine cocktail-matured MoDCs that are unable to produce IL-12 (29) are readily able to induce Th1 responses in these patients (27, 29, 30). Also, the use of BM-DC from IL-12-deficient mice for vaccination against Leishmania infection indicated that the development of Th1 responses relied on an undetermined source of IL-12 production by the recipient mice, not the injected DCs (31). These findings force researchers to question the common belief that injected vaccine MoDCs do provide signals 1, 2, and 3 for Th1 priming. In fact, it has been proposed that CCR7-dependent MoDC migration and production of IL-12 are mutually exclusive functions (32). We have shown before that injected BM-DCs reaching the draining lymph node lack IL-12 production, and they rather induce cytokine production by host endogenous DCs (33), suggesting transfer of Th1-instructing information to endogenous DCs. Indeed, cooperation between $\mathrm{pDCs}$ and $\mathrm{cDC}$ subsets can improve antiviral $\mathrm{CD}^{+} \mathrm{T}$ cell immune responses, although IL-12 production was not investigated (34). Although there is ample evidence for endogenous bystander IL-12 production, the endogenous IL-12 source for Th1 induction has not been identified.

In this study, we generated a chimeric situation by injection of different gene-modified BM-DCs into different strains of gene-modified recipient mice. This allowed us to identify the separate functional contributions of injected versus endogenous DCs for Th1 polarization. We identified the cellular source of IL-12p70 production after s.c. BM-DC vaccination as endogenous resident XCR $1^{+}$bystander DCs in the skin draining lymph nodes. DC-DC and DC-T cell interaction studies revealed a time course of Th0 priming by injected BM-DCs, followed by antigen transfer and bystander activation of the IL- $12^{+} \mathrm{XCR} 1^{+}$ bystander DCs by BM-DCs, and finally IL- $12^{+} \mathrm{XCR} 1^{+}$bystander DC interactions for Th1 induction. Transcriptional profiling of the bystander DCs underscores their Th1 polarization potential. This study shows that DC vaccination requires the bystander activation of endogenous DCs for Th1 priming.

\section{Results}

$I L-12 p 70$ production by injected OVA-loaded BM-DCS is not required for Th1 polarization. To address whether the injected DCs are capable of providing all 3 signals for the priming, proliferation, and polarization of antigen-specific $\mathrm{CD}^{+} \mathrm{T}$ cells toward a Th1 response, we used BM-DCs as a source of MoDCs (17). Following the i.v. injection of CellTrace Violet-labeled (CTV-labeled) OT-II ${ }^{+}$Thy $1.1^{+}$cells in mice with a Thy1.2 background, OVA-loaded BM-DCs that were matured with LPS (OVA-LPS/DC) were injected into footpads to induce a Th1 response in the popliteal skin draining lymph node. BM-DCs were detected by their fluorescence label between 24 and 72 hours after injection but disappeared after 6 days (Supplemental Figure 1; supplemental material available online with this article; https://doi.org/10.1172/jci.insight.135143DS1). T cell proliferation and cytokine production were analyzed at day 6 (d6) (Figure 1A). We tested whether migration of the injected BM-DCs to the popliteal lymph nodes is required for antigen presentation. OVA-LPS/DCs were generated from migration-deficient $\mathrm{Cr}_{\mathrm{C}} 7^{-1}$ mice, as shown before (35). The results indicate that the lymph nodes were enlarged by the $C c r 7^{-1-}$ BM-DC injection, similar to $C c r 7^{+/+}$BM-DC injections, clearly indicating inflammation in the lymph node. However, no T cell expansion was observed in $C c r 7^{-/} \mathrm{BM}-\mathrm{DC}$ injected animals (Figure 1B). As a control, OT-II ${ }^{+}$Thy $1.1^{+} \mathrm{CD} 4^{+} \mathrm{T}$ cells did not expand without BM-DC injection, and lymph node size did not increase (Figure 1B). This indicated that the migration of injected $\mathrm{BM}-\mathrm{DC}$ s to the lymph node is required for $\mathrm{T}$ cell expansion. It also excludes that nonmigratory BM-DCs hand over antigen at the injection site to endogenous DCs that take over or add to the $\mathrm{T}$ cell priming.

To test whether the injected BM-DCs also directly provide the Th1 polarizing IL-12 signal, LPS-matured OVA-loaded BM-DCs were injected and $\mathrm{T}$ cell cytokines were measured by intracellular FACS analysis. OT-II ${ }^{+}$Thy $1.1^{+} \mathrm{CD}^{+} \mathrm{T}$ cells induced IFN- $\gamma$ and IL-2 production, indicative of a Th1 polarization (Figure 1C). Surprisingly, a similar cytokine response was observed when the injected OVA-LPS/DCs were generated from mice lacking the p35 subunit (Il12a) of the IL-12p70 heterodimer. No significant reduction in lymph 
A

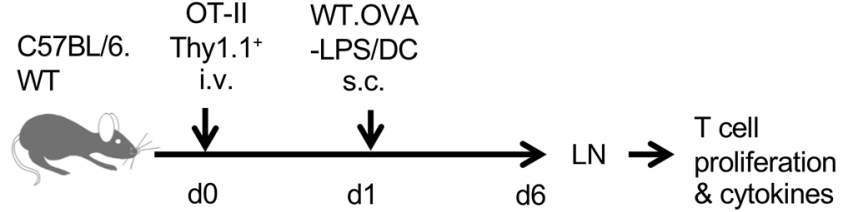

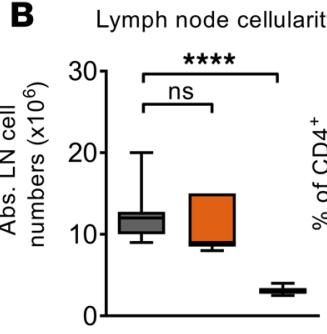

C

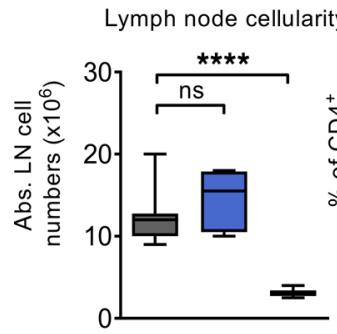

D Lymph node cellularity

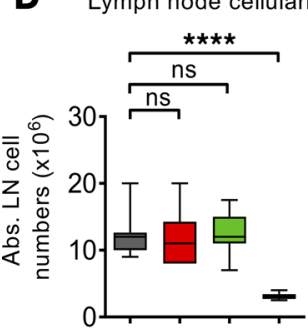

OT-II frequency

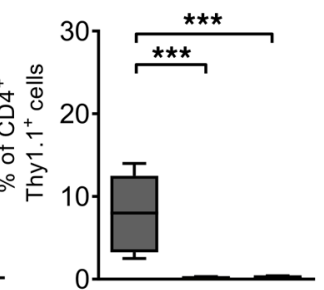

WT.OVA-LPS/DC

$\rightarrow$ WT recipient

$C c r 7^{-1-}$.OVA-LPS/DC

$\rightarrow$ WT recipient

$T$ cells only

->WT recipient

Frequency of cytokine producing OT-II cells
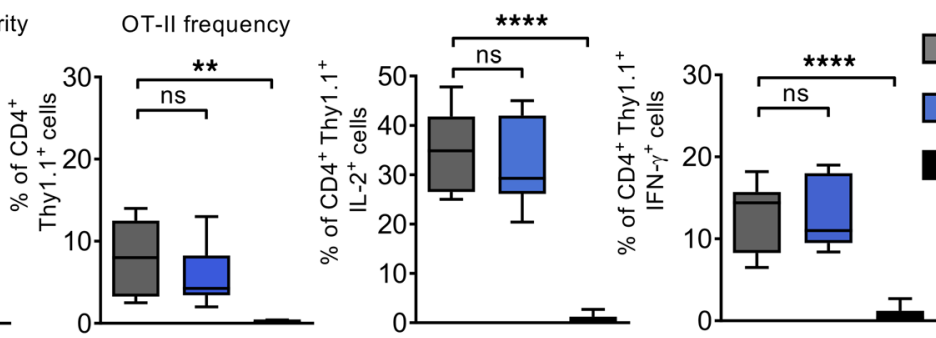

WT.OVA-LPS/DC

->WT recipient

I/12a $\%$.OVA-LPS/DC

->WT recipient

$T$ cells only

$\rightarrow$ WT recipient
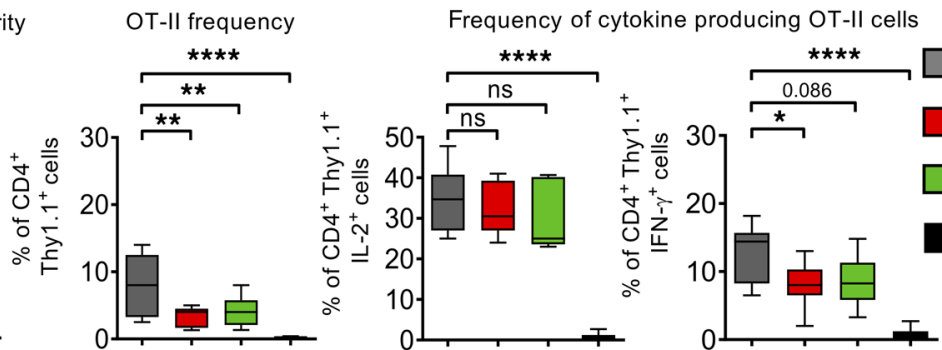

WT.OVA-LPS/DC

$\rightarrow$ WT recipient

WT.OVA-LPS/DC

$\rightarrow 1112 a^{-\%}$ recipient

II12a--OVA-LPS/DC

$\rightarrow 1112 a^{-1-}$ recipient

$T$ cells only

$\rightarrow$ WT recipient

Figure 1. Efficient DC vaccination IL-12p70 production by endogenous DCs rather than injected BM-DCs. (A) OT-II+Thy1.1+ $\mathrm{T}$ cell priming analyzed after 6 days of OVA-loaded $(10 \mu \mathrm{M})$, LPS-matured $(0.5 \mu \mathrm{g} / \mathrm{mL})$ BM-DC (WT.OVA-LPS/DC) s.c. footpad injection. (B) Graphs comparing lymph node cell counts and frequency of injected OT-II+Thy1.1+CD4+ T cells after s.c. injection of WT.OVA-LPS/DC (gray bars) or Ccr7 $7^{-1-}$ OVA-LPS/DC (orange bars) compared with $\mathrm{T}$ cell injection alone (black bars). (C) Graphs comparing lymph node cell counts, frequency of injected OT-II+Thy1.1+CD4+ $4^{+}$cells, and percentage of the cytokine-producing cells after s.c. injection of WT.OVA-LPS/DC (gray bars) or /112a-/- OVA-LPS/DC (blue bars) both into C57BL/6 WT recipient mice compared with T cell injection alone (black bars). (D) Graphs comparing lymph node cell counts, frequency of injected OT-II+Thy1.1. CD4+ T cells, and percentage of the cytokine=producing cells after s.c. injection of WT.OVA-LPS/DC into WT recipient mice (gray bar), WT.OVA-LPS/DC into $1 / 12 a^{-/-}$recipient mice (red bars), and $/ 112 a^{-/-}$OVA-LPS/DC into $/ 112 a^{-/-}$recipient mice (green bars), compared with T cell injection alone (black bars). Data are representative of 3 independent experiments analyzing at least 5 mice per group. One-way ANOVA with multiple comparisons and Tukey's post hoc test; ${ }^{*} P<0.05,{ }^{* *} P<0.01,{ }^{* *} P<0.005,{ }^{* * * *} P<0.001$.

node cellularity and in OT-II ${ }^{+}$Thy $1.1^{+} \mathrm{CD} 4^{+} \mathrm{T}$ cell expansion (Figure 1C) or in IFN- $\gamma$ and IL-2 production (Figure 1C) was observed following Il12 $a^{-/-}$BM-DC injection. Together, our data are in agreement with previous reports $(27,31)$ indicating that IL-12p70 production by injected BM-DCs is not required for CD4 $4^{+} \mathrm{T}$ cell priming and Th1 polarization. It remains to be determined whether another cellular source can provide IL-12 to direct Th1 polarization.

IL-12p70 production by endogenous cells is required for Th1 polarization. Since IL-12p70 production by the injected BM-DCs was not needed to generate a Th1 response, we tested whether an endogenous cellular

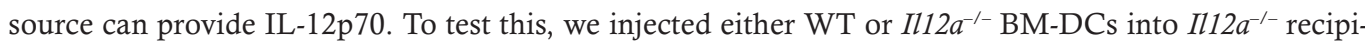
ent mice after OT-II+Thy $1.1^{+}$injection, as described before. On $\mathrm{d} 6$, lymph node cellularity increased in all groups, similar to when using WT recipients. However, the percentage OT-II ${ }^{+} \mathrm{Thy} 1.1^{+} \mathrm{CD} 4^{+} \mathrm{T}$ cells was significantly reduced in $I l 12 a^{-/-}$recipient mice (Figure 1D). This may indicate that BM-DC-derived 
p35 could contribute directly or indirectly to the general expansion of antigen-specific $\mathrm{T}$ cells in the draining lymph node.

Analyzing the cytokine production of the OT-II cells in the same experimental settings, the frequency of IL-2-producing T cells remained unchanged, while the percentage of IFN- $\gamma$ producers significantly declined (Figure 1D). These data indicate that, in the absence of endogenous IL-12p35, the priming of $\mathrm{T}$ cells by injected BM-DCs remains intact and the antigen-specific CD4 ${ }^{+} \mathrm{T}$ cells develop into an intermediate IL-2 ${ }^{+}$IFN- $\gamma^{-}$Th0 phenotype. The reduced frequency of OT-II T cells in IL-12p35-deficient mice also indicates that the function of IL-12 goes beyond Th1 instruction. Polarization into Th1 cells requires an endogenous cellular source for IL-12p70. While BM-DC-derived IL-12p35 does not play a role for Th1 polarization, it may contribute by indirect effects on inflammation and $\mathrm{T}$ cell expansion.

MHC-II ${ }^{\text {hi }} \mathrm{CD} 103^{+} \mathrm{cDC1}$ are the major source of IL-12p40 in the draining lymph node. To identify the endogenous cell subset responsible for the production of the third signal for Th1 response, we made use of Yet40 mice that express YFP under control of the promoter of the p40 subunit of IL-12 (36). In the skin draining lymph nodes of untreated healthy mice, we noticed that the only IL-12p40-YFP-producing cells were CD11 $\mathrm{c}^{\text {int }} \mathrm{MHC}-\mathrm{II}^{\mathrm{hi}}$ cells that also expressed CCR7 (Figure 2A). The IL-12p40+ population corresponds to the CCR $7^{+}$tolerogenic steady state migratory DCs $(5,37)$. The constitutive expression of IL-12p 40 at the steady state was observed previously, and it was suggested that the formation of $\mathrm{p} 40$ homodimers has an antagonistic effect on IL-12p70 (38). A significant increase in IL-12p40-YFP production above this basal level was considered as proinflammatory IL-12p40, required for the production of Th1-inducing IL-12p70.

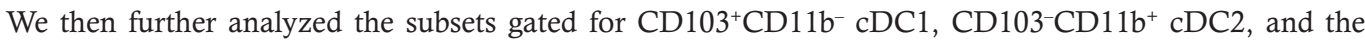
double negative (DN) DCs for both markers within the CD11 $\mathrm{c}^{\mathrm{int}} \mathrm{MHC}-\mathrm{II}^{\mathrm{hi}}$ population (Figure $2 \mathrm{~B}$ ). Within the $\mathrm{CD} 11 \mathrm{c}^{\mathrm{int}} \mathrm{MHC}-\mathrm{II}^{\mathrm{hi}} \mathrm{DC}$ gate, the $\mathrm{CD} 103^{+} \mathrm{CD} 11 \mathrm{~b}^{-} \mathrm{cDC} 1$ subset was the main source for steady state IL12-p40-YFP production (Figure 2D, right panel).

To test for bystander production of IL-12 after DC vaccination, eFluor 670-labeled/LPS-matured BM-DCs were injected s.c. into the flank or footpad of Yet40 mice, and the YFP production in the inguinal or popliteal lymph nodes, respectively, was analyzed after 24, 48, and 72 hours. eFluor 670 labeling of injected BM-DCs was used to exclude them from the analyses of the endogenous DC subsets. All 3 DC subsets increased their frequency in the draining lymph nodes in response to the injection-induced inflammation (Figure 2, C-E, left panels). Only the $\mathrm{CD} 103^{+} \mathrm{XCR} 1^{+} \mathrm{CD} 11 \mathrm{~b}^{-} \mathrm{cDC} 1$ subset showed a significant increase in IL-12p40-YFP production in a time-dependent fashion (Figure 2D, right panel). No YFP production was observed by $\mathrm{CD} 11 \mathrm{~b}^{+} \mathrm{CD} 103^{-} \mathrm{cDC} 2 \mathrm{~s}$ (Figure $2 \mathrm{C}$, right panel). CD11b ${ }^{-} \mathrm{CD} 103^{-}$(DN) DCs showed substantial YFP production under steady-state conditions, which significantly dropped 24 and 48 hours after BM-DC injection (Figure 2E, right panel). Although only IL-12p40 was analyzed here, these data suggest that $\mathrm{CD} 103^{+} \mathrm{cDC} 1 \mathrm{~s}$ were the major candidate for bystander IL-12p70 production for Th1 polarization after BM-DC injection.

CCR7-independent resident DCS provide the third signal for Th1 polarization and contribute to antigen presentation. The CD11 $\mathrm{c}^{\mathrm{int}} \mathrm{MHC}-\mathrm{II}^{\mathrm{hi}}$ population is composed mainly of migratory DCs, as described before (5). However, resident CD11 chi MHC-II ${ }^{\text {int }}$ DCs increase their MHC-II expression when they mature and, thus, enter this gate. To identify whether the $\mathrm{CD} 11 \mathrm{c}^{\mathrm{int}} \mathrm{MHC}-\mathrm{II}^{\mathrm{hi}}$ endogenous DC subset required for Th1 polarization after BM-DC injection is a migratory or a resident one, we used $C \mathrm{cr} 7^{-1-}$ recipient mice that lack endogenous migratory DCs. These mice were injected with BM-DCs generated from $I l 12 a^{-1-}$ animals. While the lymph node cellularity decreased, the frequency of OT-II cells was increased (Figure 3A), but no significant reduction in the frequency of IL- 2 and only a trend for IFN- $\gamma$-producing T cells was observed when compared with the WT recipients injected with $I l 12 a^{-/-}$DCs (Figure 3A). This indicates that endogenous $\mathrm{CCR} 7^{+}$migratory DCs of the recipient mouse do not significantly contribute to Th1 polarization after BM-DC injection. On the other hand, injecting $I l 12 a^{-1-}$ BM-DCs into $I l 12 a^{-1-} \mathrm{Cr} 7^{-1-}$ recipient mice showed comparable values for lymph node cellularity and OT-II frequency compared with injection of $I 112 a^{-1}$ BM-DCs into WT mice, but there was a strong drop in IL-2-producing T cells and basically a complete loss of IFN- $\gamma$ producers, indicating a residual Th0 response (Figure $3 \mathrm{~A}$ ). These findings point to a resident CCR7- DC subset as the main producers of IL-12 required for Th1 polarization.

The question remained whether the endogenous DCs only deliver IL-12 or also contribute to antigen presentation. To test this, we used MHC-II-deficient mice as recipients and injected WT BM-DCs. Due to a general lack of $\mathrm{CD} 4^{+} \mathrm{T}$ cells in these mice, the lymph node cellularity was significantly reduced compared with WT recipient mice (Figure 3A). Similar to the IL-12 deficiency of recipient mice, the lack of MHC-II 
A
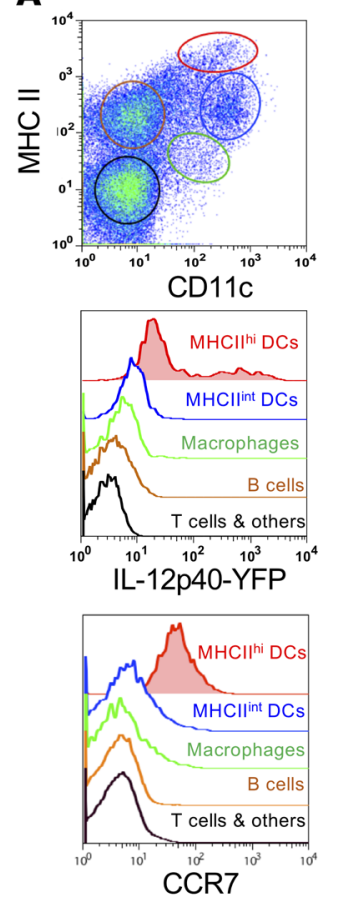

B

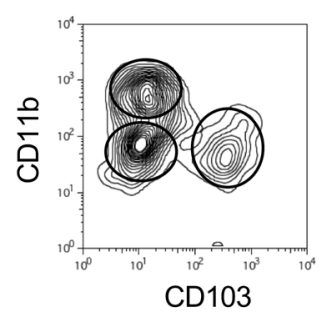

C

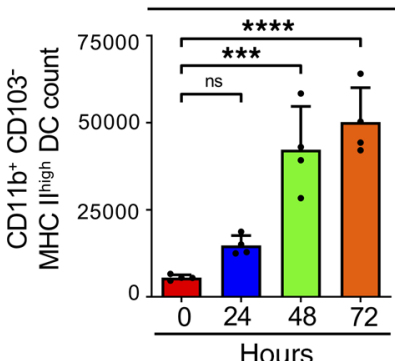

CD103- CD11 $\mathrm{b}^{+} \mathrm{MHC}$ II $^{\text {high }} \mathrm{DCs}$
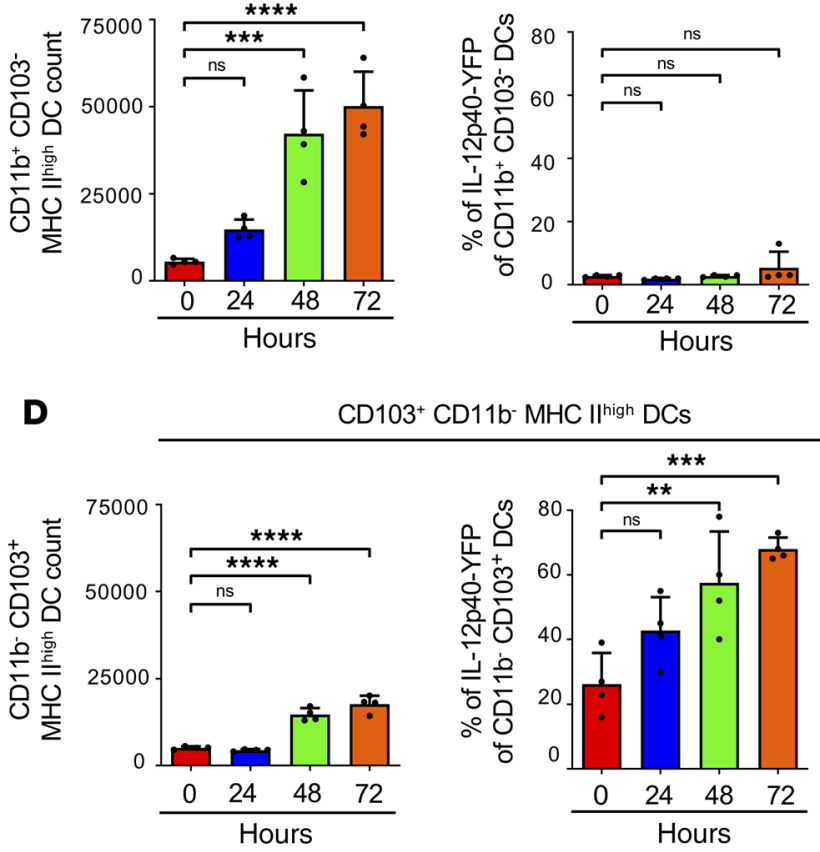

D103+ CD11b-MHC II high DCs

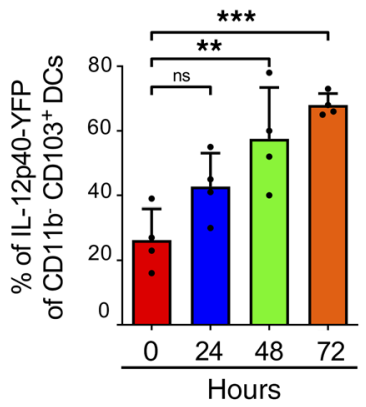

$\mathbf{E}$

CD103- CD11b-MHC II high (DN) DCs

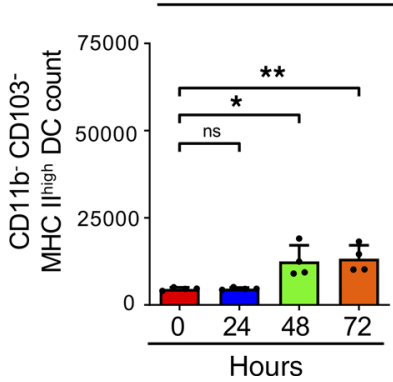

Figure 2. Endogenous MHC-II ${ }^{\text {iCD103 }}{ }^{+}$XCR1+Langerin $+C D 11 b^{10}$ DCs are the main producers of IL-12p40 after DC vaccination. (A) Representative flow cytometry plot of popliteal lymph nodes subpopulations gated based on their expression of CD11C and MHC-II in a Yet40 reporter mouse (upper panel) and histogram plots of IL-12p40-YFP production and CCR7 expression of each subpopulation (lower panels). (B) Gating strategy to identify CD11C $\mathrm{C}^{+} \mathrm{MHC}-\mathrm{II}^{\text {hi }} \mathrm{DC}$ subsets. (C-E) Graphs showing absolute counts of CD11c ${ }^{+}$MHC-II ${ }^{\text {hi }}$ DCs (left panels) and percentage of IL-12p40-YFP producing cells from CD11b+ dermal DCs (C) CD103+ dermal DCs (D) and DN DCs (E) (right panels) after s.c. injection of WT.LPS/DC into Yet40 recipient mice (24-, 48-, 72-hour time points). Data are representative of 3 independent experiments analyzing at least 5 mice per group. One-way ANOVA with multiple comparisons and Tukey's post hoc test; ${ }^{*} P<0.05,{ }^{* *} P<0.01,{ }^{* *} P<0.005,{ }^{* * *} P<0.001$.

antigen presentation also dramatically affected OT-II frequencies and reduced the frequencies of IL-2 ${ }^{+}$and IFN $-\gamma^{+} \mathrm{T}$ cells (Figure $3 \mathrm{~A}$ ). These results suggest that endogenous resident DCs substantially contribute to antigen presentation.

To this point, the results indicate that endogenous lymph node-resident CCR7-CD103+ $\mathrm{cDC} 1 \mathrm{~s}$ represent the bystander DC population that is producing IL-12 for the Th1 polarization of OT-II cells. The bystander $\mathrm{CDC} 1$ also substantially contributes to antigen presentation, suggesting an OVA antigen transfer from the injected BM-DCs to the bystander $\mathrm{CDC} 1 \mathrm{~s}$.

$X C R 1^{+} C D 103^{+} C D C 1$ provide the third signal for Th1 induction after BMDC injection. So far, all evidence points to $\mathrm{CCR} 7-\mathrm{CD} 103^{+} \mathrm{CDC} 1 \mathrm{~s}$ as endogenous IL-12 producers for Th1 polarization in the BM-DC injection setup. To conclusively show the critical role of $\mathrm{CDC} 1 \mathrm{~s}$ for Th1 polarization, we used XCR1-DTRVenus mice, where the $\mathrm{XCR} 1^{+} \mathrm{CD} 103^{+} \mathrm{CDC} 1$ subset can be conditionally depleted by diphtheria toxin (DTX) injection (39). The mice were injected with OT-II'Thy1.1 $1^{+}$cells followed by $I 112 a^{-1}$ OVA-LPS/ $\mathrm{DC}$ injection as described before; then XCR $1^{+} \mathrm{DC}$ s were depleted using DTX. In these mice, there was a 
A Lymph node cellularity
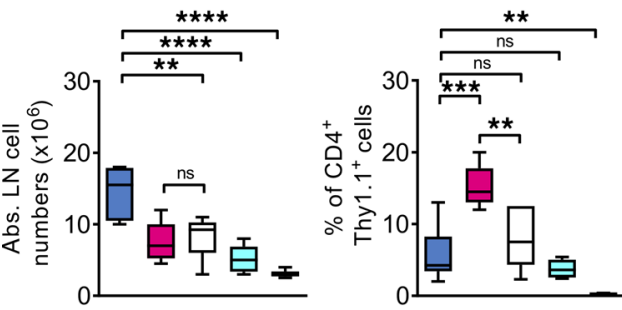

B Lymph node cellularity

OT-II frequency
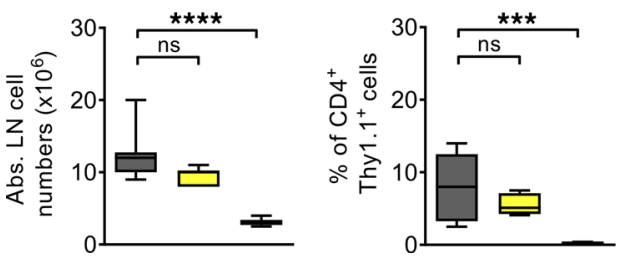

OT-II frequency

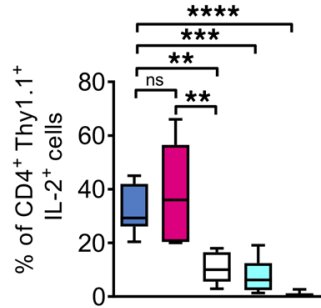

Frequency of cytokine producing OT-II cells

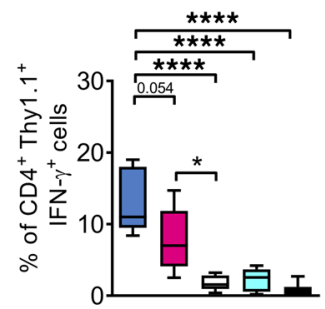

$1112 a^{-1-}$. OVA-LPS/DC

$\rightarrow$ WT recipient

$1 / 12 a^{-1-}$. OVA-LPS/DC

$\rightarrow \mathrm{Cr}^{-1-}$ recipient

$1 / 12 a^{-1-}$.OVA-LPS/DC

$\rightarrow 1112 a^{-1} \cdot C c r 7^{-1-}$ recipient

WT.OVA-LPS/DC

$\rightarrow \mathrm{MHCl}^{-1-}$ recipient

$T$ cells only

->WT recipient

Frequency of cytokine producing OT-II cells
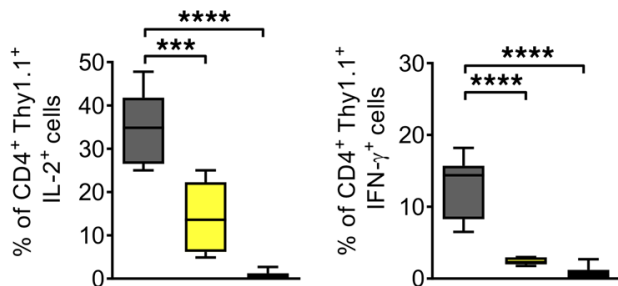

WT.OVA-LPS/DC

$\rightarrow$ WT recipient

II12a $a^{-/}$.OVA-LPS/DC

$\rightarrow$ XCR1-DTR-venus

recipient

$T$ cells only

->WT recipient

Figure 3. Endogenous $C D 103^{+} X C R 1^{+}$resident $D C s$ are required for antigen presentation and Th1 polarization. (A) Graphs comparing lymph node cell counts, frequency of injected OT-II+Thy1.1+CD4+ T cells, and percentage of the cytokine-producing cells after s.c. injection of $/ 112 a^{-1-}$ OVA-LPS/DC into

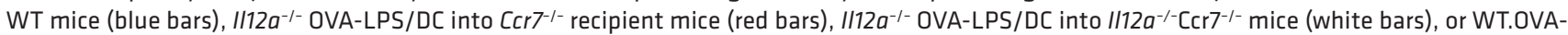
LPS/DC into MHC-II-- (turquoise bars) recipient mice compared with T cell injection alone (black bars). (B) Graphs comparing lymph node cell counts, frequency of injected OT-II+Thy $1.1^{+}$CD4 ${ }^{+}$T cells, and percentage of the cytokine-producing cells after s.c. injection of WT.OVA-LPS/DC into WT recipient mice (gray bar) or $1 / 12 a^{-1-}$ OVA-LPS/DC into XCR1-DTR-Venus recipient mice (yellow bars) compared with T cell injection alone (black bars). Data are representative of 2 independent experiments analyzing at least 5 mice per group. One-way ANOVA with multiple comparisons and Tukey's post hoc test; ${ }^{*} P<0.05,{ }^{* *} P<0.01,{ }^{* *} P<0.005,{ }^{* * *} P<0.001$.

slight reduction in lymph node cellularity at $\mathrm{d} 6$ after BM-DC injection, while CD4 ${ }^{+} \mathrm{OT}-\mathrm{II}^{+} \mathrm{Thy} 1.1^{+} \mathrm{T}$ cells expanded similar to WT mice (Figure 3B). The production of IL-2, however, was significantly reduced, indicating an impairment in T cell priming, and IFN- $\gamma$ production was completely lost (Figure 3B). These results conclusively show the requirement of $\mathrm{XCR} 1{ }^{+} \mathrm{CD} 103^{+} \mathrm{CDC} 1 \mathrm{~s}$ as the critical bystander DC subset to allow Th1 polarization after BM-DC vaccination.

$Y F P^{+}$endogenous DCs communicate with injected BM-DCs at 48-72 hours. The contribution of bystander DCs to antigen presentation suggests antigen transfer from injected BM-DCs that would require their interaction in the draining lymph nodes. One possibility of DC-DC interaction leading to bystander activation is that LPS bound to TLR4 on the migrated BM-DCs is presented to other DCs in the lymph node. In vitro data indicate that LPS-matured BM-DCs can activate cocultured immature BM-DCs to secrete IL-12p40, while CpG-matured BM-DCs were unable to show this effect (Supplemental Figure 2). These data suggest that LPS remains bound to surface TLR4 and can be presented to bystander DCs, while CpG seems to be efficiently internalized by DEC-205/CD205 to bind TLR9 within intracellular vesicles (40). Since DEC-205 is expressed specifically on XCR1 ${ }^{+}$cDC1s, as detected by the NLDC-145 antibody (41), we hypothesized that injection of CpG-matured BM-DCs should abrogate bystander Th1 polarization in vivo. However, the use of CpG-matured, OVA-loaded BM-DCs (OVA-CpG/DC) provoked a similar lymph node swelling, OT-II ${ }^{+}$Thy $1.1^{+} \mathrm{CD} 4^{+} \mathrm{T}$ cell expansion and Th1 frequencies, as observed after OVA-LPS/ DC injection, while the frequency of IL-2+ OT-II cells was slightly reduced (Supplemental Figure 3 ). These data indicate that injected BM-DCs possess additional mechanisms of bystander DC activation beyond the presentation of surface-bound pathogen.

To study potential DC-DC and DC-T cell communication patterns in the lymph node, OT-II ${ }^{+}$Thy $1.1^{+}$ $\mathrm{T}$ cells were injected i.v. and CTV-labeled LPS-BM-DCs generated from $\mathrm{Ill}_{2} \mathrm{a}^{-1-}$ mice were injected the next day into the footpads of the mice. The interaction between the injected T cells, BM-DCs, and YFP ${ }^{+}$ endogenous DCs was analyzed 24, 48, and 72 hours after their injection within the T cell area of popliteal draining lymph nodes by confocal microscopy. We observed distinct clusters of OT-II'Thy $1.1^{+}$cells with CTV-labeled BM-DCs within the T cell area at the 24-hour time point, as described before $(42,43)$. During this period, T cells form long-lasting stable conjugates with DCs (Figure 4A). At 48 hours, the presence of CTV-labeled BM-DCs peaked in the lymph nodes and declined thereafter (Figure 4B). OT-II ${ }^{+}$Thy $1.1^{+}$ 
T cell expansion was observed peaking at 72 hours (Figure 4B). The number of IL-12p40+ cells remained constant, showing only a trend of higher frequencies at 48 hours (Figure 4B). We then analyzed the change in relative distance of $\mathrm{YFP}^{+}$endogenous DCs to the injected CTV-labeled BM-DCs and compared it with the relative distance between the total $\mathrm{XCR} 1^{+}$endogenous $\mathrm{CDC} 1 \mathrm{~s}$, which includes the presumed bystander-activated $\mathrm{CD} 103^{+} \mathrm{XCR} 1^{+} \mathrm{CDC} 1 \mathrm{~s}$ and the injected BM-DCs; as a control, the distance between the unrelated $\mathrm{CD} 11 \mathrm{~b}^{+} \mathrm{cDC} 2 \mathrm{~s}$ and the injected BM-DCs was measured. The relative distance between $\mathrm{CD} 11 \mathrm{~b}^{+}$and total XCR $1^{+}$endogenous DCs to injected BM-DCs showed no signs of approximation but rather increased at the 48-hour and 72-hour time points when compared with the 24-hour time point (Figure 4C, green arrow directions). This is attributed to their random movement in the lymph node that gets enlarged at the 48-hour and 72-hour time point. When the relative distance between the specifically IL-12p40-producing $\mathrm{YFP}^{+}$endogenous XCR1 ${ }^{+} \mathrm{cDC} 1 \mathrm{~s}$ and the injected BM-DCs was measured, a significant reduction of their distance at both 48-hour and 72-hour time points was observed when compared with 24 hours (Figure 4C, green arrow directions). These data indicate that injected BM-DCs communicate with endogenous XCR $1^{+} \mathrm{IL}-12 \mathrm{p} 40^{+} \mathrm{cDC} 1 \mathrm{~s}$ in the lymph node paracortex areas to mediate the observed Th1 induction between 48 and 72 hours.

Cognate T cells show early communication with injected BM-DCs and later with $Y F P^{+}$endogenous DCs. If the $\mathrm{YFP}^{+}$endogenous DCs are indeed receiving signals from the injected BM-DCs at later time points, we expected them to provide the IL-12 signal to T cells later, during the T cell expansion phase. This was already indicated by the fact that IL-12p40-YFP peaked at 72 hours (Figure 2C), indicating its requirement later in the $\mathrm{T}$ cell expansion phase. To confirm that, we measured the relative distances of OT-II+Thy $1.1^{+} \mathrm{T}$ cells to either injected BM-DCs or $\mathrm{YFP}^{+}$endogenous DCs. The relative distance between T cells and BM-DCs showed a tendency to increase after 48 hours and significantly at 72 hours compared with the 24-hour time point, possibly due to the increase in lymph node size or the termination of antigen presentation. On the other hand, the relative distance between $\mathrm{T}$ cells and $\mathrm{YFP}^{+}$ endogenous DCs was significantly reduced after 48 hours and 72 hours, as compared with the 24-hour time point (Figure 4C).

These findings suggest a coordinated timing of interactions between 2 DC types and the cognate $\mathrm{T}$ cells as sequential steps - first, between the injected BM-DCs and cognate T cells; second, the BM-DCs with $\mathrm{YFP}^{+} \mathrm{XCR} 1^{+}$endogenous bystander $\mathrm{CDC} 1 \mathrm{~s}$; and third, $\mathrm{YFP}^{+} \mathrm{XCR} 1^{+} \mathrm{IL}-12 \mathrm{p} 40^{+}$endogenous bystander cDC1s with the primed Th0 cells for further polarization into Th1 cells (Figure 4D).

Transcriptional profiling of the endogenous XCR $1^{+}$bystander $C D C 1$ s. Since the IL- $12^{+} \mathrm{CD} 103^{+} \mathrm{XCR} 1^{+}$bystander $\mathrm{CDC} 1$ are recruited from the lymph node-resident population but IL-12p40+ cells are only detectable among MHC-II ${ }^{\text {hi }}$-expressing DCs (Figure 2C), the immature resident bystander DC population underwent maturation/activation by the DC vaccination process. To study the transcriptional changes occurring in the endogenous matured bystander $\mathrm{cDC} 1$, we sorted the $\mathrm{MHC}-\mathrm{II}^{\text {hi }} \mathrm{CD} 11 \mathrm{c}^{+}$lymph node DC subsets before (naive mice) and 48 hours after BM-DC injection. Therefore, we could compare MHC- $-I^{\text {hiX XCR } 1^{+} C D 11 b}$

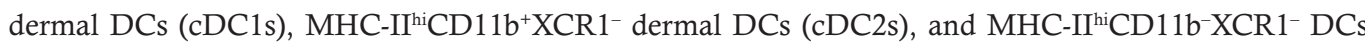
$(\mathrm{DN})$ at steady-state and 48 hours after LPS-BM-DC injection. Also, the appearance of MHC-II ${ }^{\mathrm{hi}} \mathrm{CD}$ $11 \mathrm{~b}^{+} \mathrm{CD} 64^{+}$Ly6 $\mathrm{C}^{\text {lo }}$ inflammation-induced MoDCs was identified, and these cells were also sorted at 48 hours. Since MoDCs could not be detected in naive mice, we were lacking a direct related control for this population. RNA sequencing (RNA-seq) was performed on 100 sorted cells from each population. Principal component analysis (PCA) segregated the samples into 3 distinct groups. Each of the $3 \mathrm{cDC}$ subsets clustered differently and shifted to a different direction after their bystander activation. MoDCs clustered close to $\mathrm{CD} 11 \mathrm{~b}^{+} \mathrm{cDC} 2 \mathrm{~s}$ and appeared further distant from bystander-activated $\mathrm{CD} 11 \mathrm{~b}^{+} \mathrm{cDC} 2 \mathrm{~s}$, indicating a close relation (Figure 5A). This may indicate that the general inflammatory situation in the size-expanded lymph node signals to all endogenous DC subsets.

Gene ontology (GO) and pathway enrichment analysis (http://geneontology.org/docs/go-enrichment-analysis/) for the differentially regulated genes in the XCR $1^{+} \mathrm{MHC}-\mathrm{II}{ }^{\mathrm{hi}} \mathrm{CDC} 1$ subset indicated a downregulation of nucleosome organization, cellular development, and cellular differentiation pathways (Figure 5B). Interestingly, genes promoting TGF- $\beta$ signaling and Treg induction, or Th 2 induction by DCs, were included in these downregulated pathways, together with genes involved in DC migration and genes that modulate the self-antigen presentation capacity of DCs (Figure 5B).

Then, we compared the genes differentially regulated in the $4 \mathrm{MHC}-\mathrm{II}^{\mathrm{hi}} \mathrm{DC}$ subsets of BM-DCinjected mice with the 3 steady state MHC-II ${ }^{\text {hi }}$ DC subsets. A total of 112 genes were significantly up- or 
A
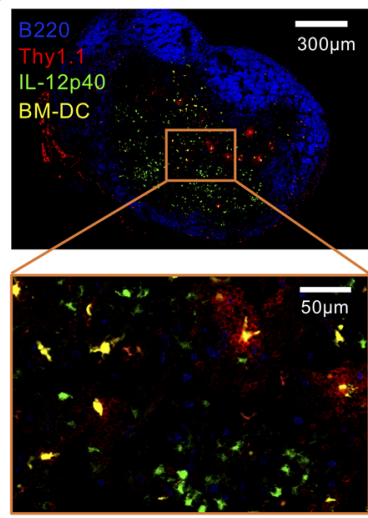

B

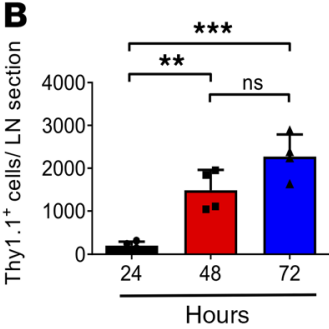

$48 \mathrm{~h}$
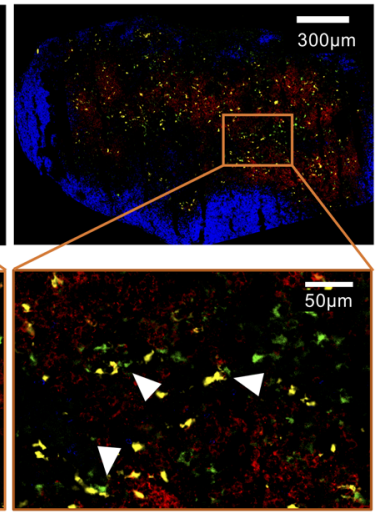
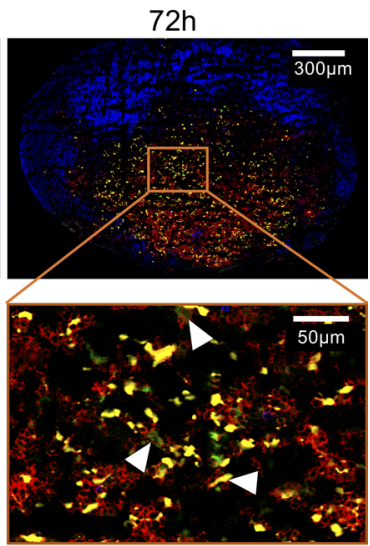

C $\quad$ CD11 $1 b^{+}$to inj-DC $\quad X C R 1^{+}$to inj-DC
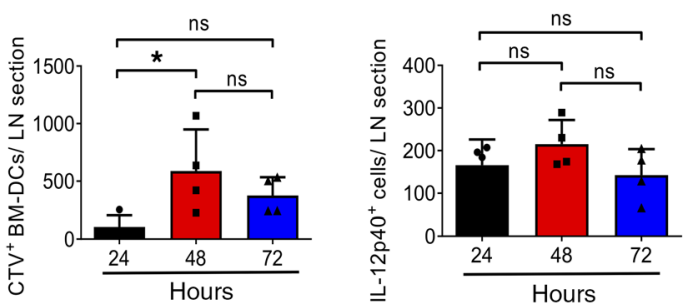

YFP $^{+}$to inj-DC $\quad$ T cells to inj-DC

T cells to YFP ${ }^{+} D C$
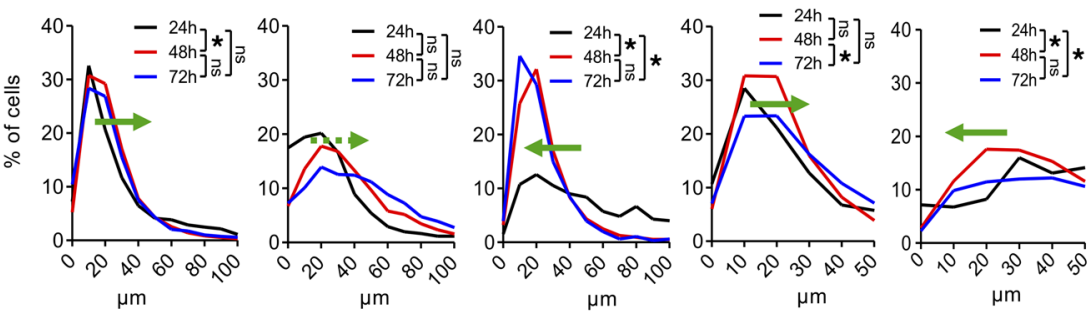

D

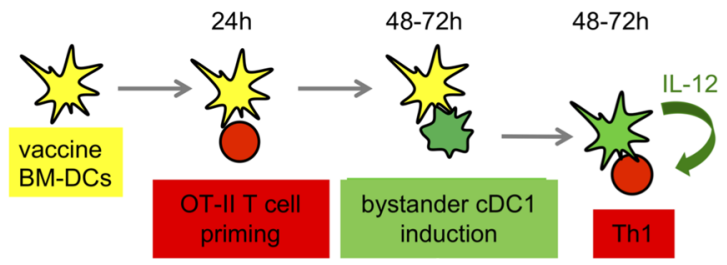

Figure 4. YFP ${ }^{+}$XR1 ${ }^{+} D C$ s interact with injected DCs and antigen-specific $T$ cells at later time points. (A) Representative immunofluorescence microscopy images of whole popliteal lymph nodes sections (upper row) and magnification of the T cell area after of OT-II+Thy1.1 $1^{+}$T cell injection (red) + CTV-labeled I/12a-/- OVA-LPS/DC s.c. injection (yellow) into IL-12p40-YFP mice (green cells) $(24,48$, or 72 hours after injection). White arrowheads point to the points of interaction between injected yellow DCs and YFP endogenous green DCs. (B) Graphs showing number of OT-II+Thy1.1+ T cells, CTV-labeled OVA-LPS/DC, and YFP+ endogenous DCs/popliteal lymph node cut 24, 58, 72 hours after DC injection. (C) Graphs showing the relative distance of CD11 $\mathrm{b}^{+}$cells, XCR1+ cells, and YFP+ ${ }^{+}$cells to $\mathrm{CTV}$-labeled OVA-LPS/DC in the peripheral lymph nodes 24,48 , or 72 hours after DC injection. Graphs showing the relative distance of OT-II+Thy1.1+ T cells to CTV-labeled OVA-LPS/DC or to YFP+ endogenous DCs in the peripheral lymph nodes 24,48 , or 72 hours after DC injection. Green arrow indicates the distance shift at 48 and 72 hours compared with 24 hours. Data are representative of 2 independent experiments analyzing at least 4 mice per group. One-way ANOVA with multiple comparisons and Tukey's post hoc test; ${ }^{* * *} P<0.0001,{ }^{* *} P<0.001,{ }^{*} P<0.05$. (D) Model about the time kinetics of cellular interactions suggested by the microscopic analyses.

downregulated in at least one of the comparisons. Distinct clusters of regulated genes were observed for each DC subset (Figure 5C). Mapk13, which is involved in cytokine production; Tmem79, which is a transmembrane protein involved in the lamellar granules secretory system and skin barrier function and which might be involved in cell-cell communication (44); and Hox4a, which is a selective transcriptional regulator, were specifically upregulated on XCR $1^{+}$bystander dermal DCs. 
A

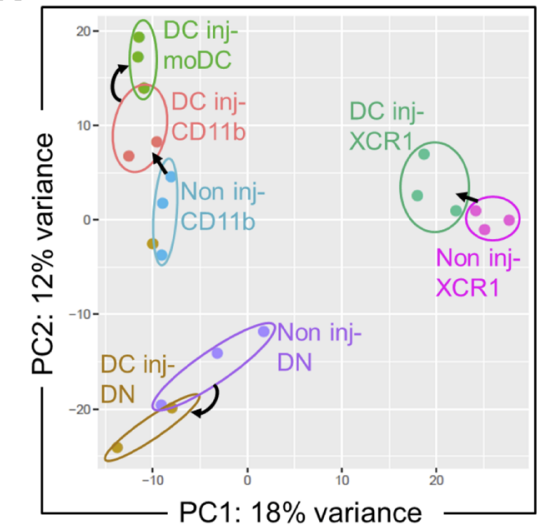

B

GO Pathway analysis of XCR1+DCs cellular developmental process $\downarrow$ cell differentiation $\downarrow$

\begin{tabular}{|l|l|}
\hline Individual genes of these pathways \\
DCs inducing Th2 and Treg \\
\begin{tabular}{|l|l|}
\hline Ahr & DC instruction of Treg induction (89) \\
\hline Aff3 & Downregulation of IL-6 and IL-12 (90) \\
\hline II3ra & Promotes Th2-polarizing DCs (91) \\
\hline II7r & DC control of T cell proliferation (92) \\
\hline Jag2 & Promotes Th2-polarizing DCs (93) \\
\hline KIf2 & Negative regulation of DCs (26) \\
\hline Pirb & Tolerogenic signals for DCs (94). \\
\hline Stat5b & Promotes Th2-polarizing DCs (95) \\
\hline Stat6 & Promotes Th2-polarizing DCs (96) \\
\hline Tgfbr2 & TGF- $\beta$ signaling and Treg induction (97) \\
\hline DC migration \\
\hline Cxcr4 & DC migration (98) \\
\hline L1cam & Transendothelial DC migration (99) \\
\hline Nfkb2 & Steady-state migration of DCs (100) \\
\hline Nr4a3 & Promotes CD103 ${ }^{+}$DC migration (101) \\
\hline DC antigen presentation \\
\hline Prdm1 & MHC II presentation on DCs (102) \\
\hline Pirb & Prevents cross-priming (94) \\
\hline Other genes \\
\hline Cd8a & DC lineage marker (103) \\
\hline Itgb7 & Gut homing (104) \\
\hline Runx1 & FIt3 ${ }^{+}$DC development (105) \\
\hline Runx2 & pDC development and migration (106) \\
\hline \hline
\end{tabular} \\
\hline
\end{tabular}

C

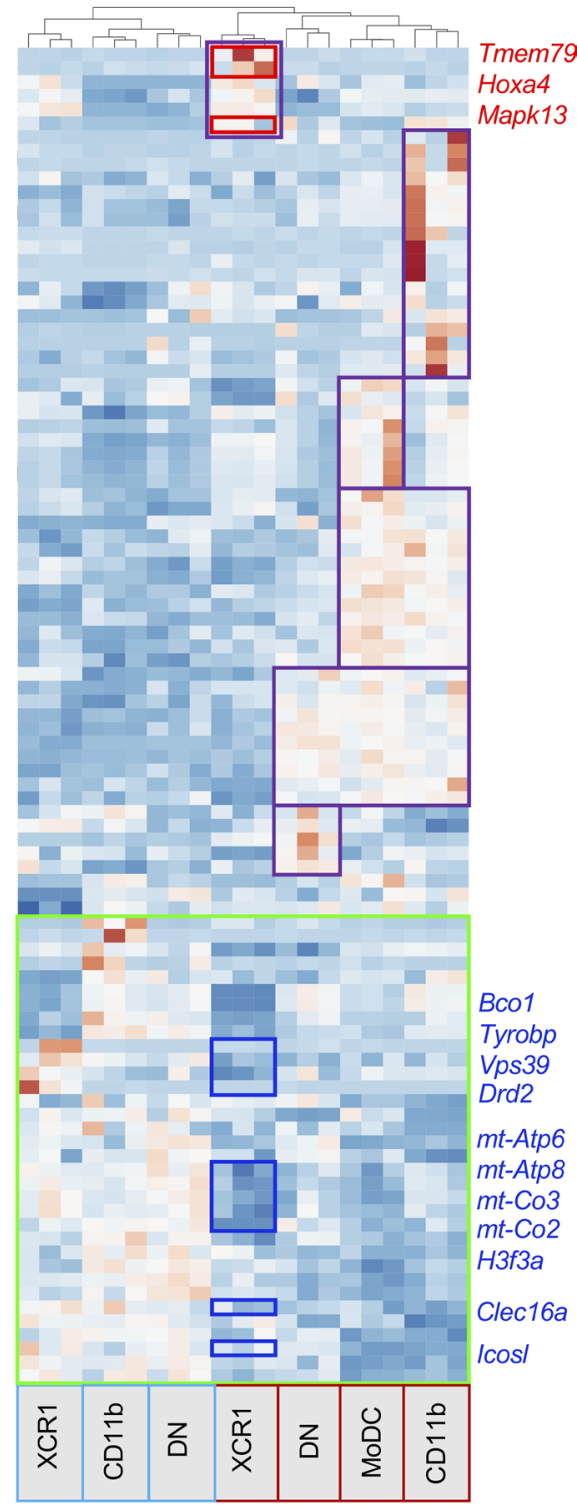

Figure 5. Endogenous migratory DC subsets have distinct transcriptional changes after bystander activation by

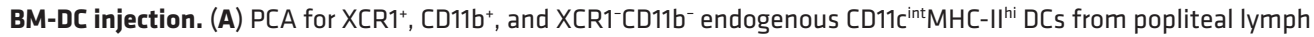
nodes before and 48 hours after immunization, and CD11 b+CD64+ ${ }^{+} y 6 C^{-}$MoDCs compared with $C D 11 b^{+} D C s$ after immunization. (B) Genes downregulated in XCR1+ DCs 48 hours $(P<0.05)$ after immunization according to the GOrilla analysis tool. Green color, down in XCR1 $1^{+}$DCs only; orange color, down in XCR1+ DCs and MoDCs (89-106). (C) Heatmap of the 112 genes that are at least 1.5-fold differentially expressed in one comparison (red, upregulated; blue, downregulated). Plotting was done using Clustvis web tool; clustering was performed using Pearson's correlation and average linkage.

Among the significantly downregulated genes were Vps39 and Clec16a, which are important for auto-lysosomal generation of self-antigens (45) and for surface expression of MHC-II molecules (46). Also, genes involved in TGF- $\beta$ signaling required for Treg induction, such as Icosl, which is important for generating Foxp $3^{+}$Tregs (47); genes promoting Th2 such as Bco1 (48); and genes blocking Th1 induction, such as Tyrobp (DAP12), which was shown to downregulate Th1 responses in intracellular infections (49-51). ATP synthesis pathways, which trigger IL-33 release and Th2 responses and subsequently block IL-12 production, were also inhibited as indicated by the downregulation of the ATP synthases mt-Atp6 and mt-Atp8 and of the cytochrome oxidases mt-Co2 and mt-Co3 $(52,53)$ (Figure 5C).

Together, our data provide a map of genes by which DC vaccine-activated MHC-II hi bystander $\mathrm{CDC} 1 \mathrm{~s}$ acquire a broad phenotype on one hand toward Th1 induction, but on the other hand, away from a tolerogenic, self-antigen presenting, Treg-inducing or Th2-inducing gene signature. 
Bystander XCR $1^{+}$cDC1s downregulate MHC-II genes and acquire IL-12-producing potential. As indicated above, self-antigen presentation appears to be downregulated in bystander XCR1 ${ }^{+} \mathrm{CDC} 1$. More detailed analyses of MHC and related genes indicated a nonsignificant reduction of several MHC-II molecules (and also the invariant chain $\mathrm{CD} 74$ ) in bystander activated $\mathrm{XCR} 1^{+} \mathrm{cDC} 1 \mathrm{~s}$ on the transcript level (Figure $6, \mathrm{~A}$ and C). Such a reduction was not observed for classical and nonclassical MHC-I molecules (Figure 6B). Contrarywise, the MHC-II protein surface expression significantly reduced at an earlier time point after BM-DC injection (24 hours) and was significantly increased again after 48 hours (Figure 6D). This suggests a transfer of MHC molecules presenting the OVA antigen from the injected BM-DCs to the endogenous bystander DCs. The transfer of antigens between DCs has been shown before (54), but the exact mechanism in our setup needs further investigations.

$I l 12 a$ and $I l 12 b$ did not appear upregulated by XCR1 ${ }^{+}$dermal DCs in the RNA-seq analysis. Nevertheless, the upregulation of $I l 12 a$ and $I l 12 b$ on bystander-activated XCR $1^{+} \mathrm{CDC} 1$ s was confirmed with real-time $\mathrm{PCR}$, and both genes were found to be specifically upregulated on the designated $\mathrm{XCR} 1^{+}$subset and not on any of the other bystander-activated DC subsets (Figure 6E). We assume that not all endogenous XCR $1^{+}$ DCs are activated to become bystander DCs, since they may carry out their tolerogenic functions (55). In addition, analyzing only 100 cells may not allow detecting enough low copy number genes, such as the IL-12a signal, and only strongly regulated genes become visible. Together, the transcriptional profiling of $\mathrm{XCR} 1^{+}$lymph node-resident bystander $\mathrm{CDC} 1 \mathrm{~s}$ is characterized by a downregulation of genes providing non-Th1 polarizing signals and the upregulation of genes such as $I l 12 a$ and $I l 12 b$ required for IL-12p70mediated Th1 polarization.

\section{Discussion}

DCs are the dominant immune cells to induce T cell priming in vivo. Also, the instruction of Th cell responses by DCs providing polarizing signals has become generally accepted. Among the Th1-instructing cytokines, IL-12p70 plays a prominent role. Here, we address whether the priming capacity and polarizing IL-12p70 signals are derived from injected vaccine DCs. We employed s.c. injection of BM-DCs into mice as a model to test different chimeric situations where injected BM-DCs and recipient mouse strains were bearing different genetic deficiencies. Our BM-DC vaccines are close to clinical studies with human MoDC vaccines, due to the fact that GM-CSF-generated BM-DCs are monocyte derived (17). The use of OVA antigen here may not allow simple extrapolation of our findings to human clinical studies using tumor-antigen pulsed MoDCs. However, our results using clear-cut genetic models to determine the source of IL-12 for Th1 induction may encourage similar investigations in human DC vaccine settings to improve vaccination success.

It has been observed before that priming of $\mathrm{CD} 8^{+} \mathrm{CTL}$ responses after virus infection or DC antitumor vaccines relies on antigen transport by migration of tissue-resident or injected DCs but that antigen presentation is largely dependent on lymph node-resident cDC1 or undefined endogenous DCs $(56,57)$. Similar1y, our data reveal that s.c. injected vaccine BM-DCs only partially contribute to antigen presentation at an early stage (24 hours), and they do not contribute to Th1 polarization. A major part of antigen presentation for Th0 induction and the entire capacity for Th1 polarization is mediated by endogenous $\mathrm{XCR} 1^{+}$resident bystander cDC1s at later time points (48-72 hours). However, BM-DCs migration to the draining lymph node is strictly required, and bystander activation for IL-12 production seems to occur in the lymph node. Our findings argue for a step-wise process of priming naive T cells into an IL-2 $2^{+}$IFN- $\gamma^{-}$Th0 phenotype by the injected DCs, followed by a communication between injected BM-DCs and XCR1 ${ }^{+}$bystander $\mathrm{CDC} 1 \mathrm{~s}$. Bystander contact includes transfer of antigen and initiation of IL-12p70 production. This period is followed by contacts of activated IL- $12 \mathrm{p} 70^{+} \mathrm{XCR} 1^{+}$bystander $\mathrm{cDC} 1 \mathrm{~s}$ with the $\mathrm{Th} 0$ cells to continue antigen presentation and conversion into Th1 polarized cells. RNA-seq allowed the identification of transcriptional changes during the conversion of lymph node-resident XCR $1^{+} \mathrm{CDC} 1 \mathrm{~s}$ into bystander-activated $\mathrm{cDC} 1 \mathrm{~s}$. Among those, DC genes known to polarize naive T cells into Treg or Th2/Th9 immune responses or to counteract IL-12 production were downregulated, while Th1 supporting genes were induced.

The optimization of DC vaccination protocols has focused mainly on enhancing the activation of generated DCs $(58,59)$, their cytokine production profile (60), and their migration capacity (61). Other studies attempted to combine the vaccine injection with adjusting the immunosuppressive milieu of the tumor microenvironment to a more immunogenic one - for example, by blocking inhibitory receptors such as PD-1/PD-L1 (62). 
A

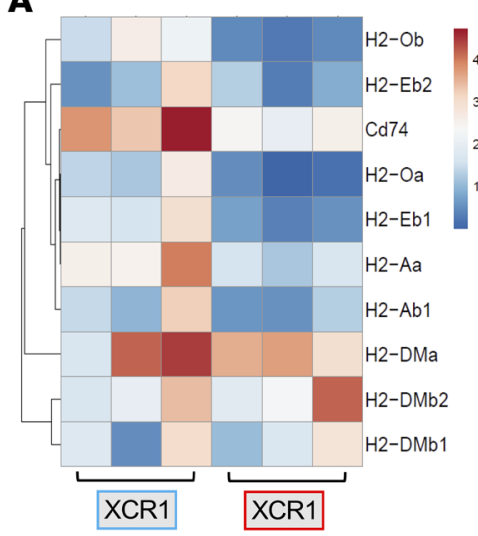

C $\mathrm{H} 2-\mathrm{Aa}$
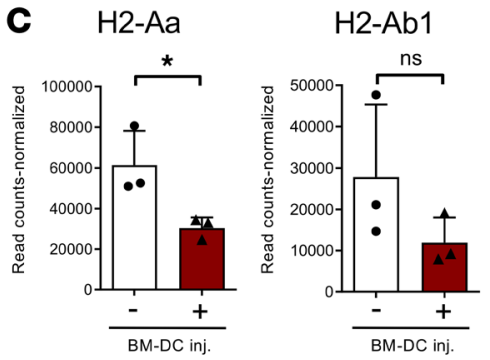

D

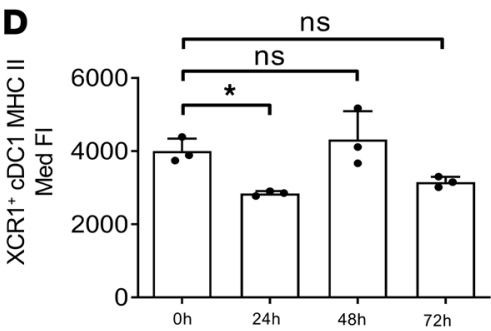

B

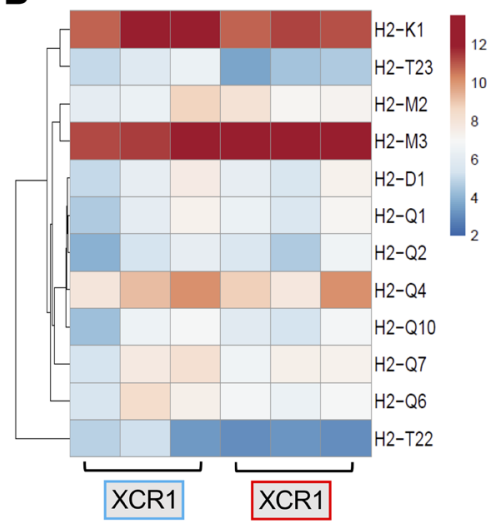

$\mathrm{H} 2-\mathrm{Aa}$

$\mathrm{H} 2-\mathrm{Ab} 1$
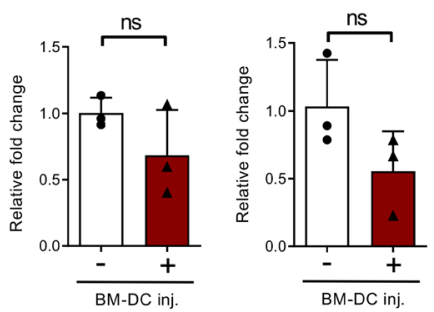

E

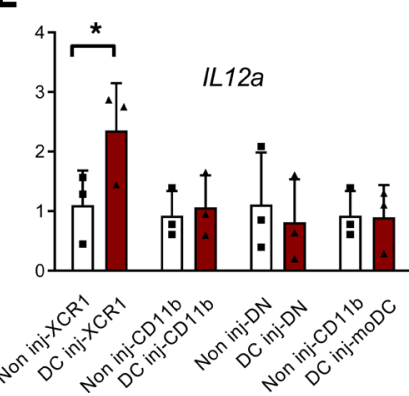

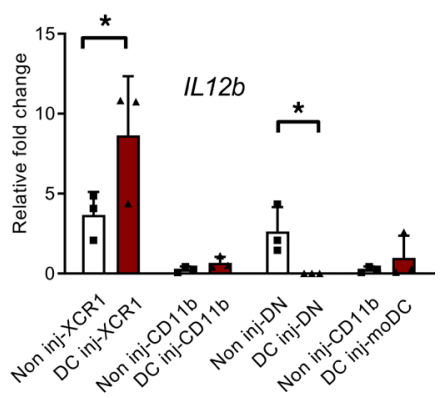

Figure 6. Loss of MHC-II but not MHC-I genes and acquisition of IL-12 production by bystander CDC1s. (A and B) Heatmap plots for the differential expression of MHC-II (left panel) and MHC-I (right panel) genes in the XCR1+CD11 ${ }^{\text {int- }}$ MHC-II ${ }^{\mathrm{hi}} \mathrm{CDC} 1$ subset before and 48 hours after immunization. (C) Normalized read counts (left panel) and relative fold change (right panel) by qPCR in the XCR1 $1^{+} \mathrm{CD} 11 \mathrm{C}^{\text {int }} \mathrm{MHC}-\mathrm{II}^{\text {hi }} \mathrm{CDC} 1$ subset before and 48 hours after immunization. (D) Median fluorescence intensity of MHC-II on XCR1+CD11 int MHC-II hi $C D C 1$ s before and 24, 48, 72 hours after BM-DC immunization. (E) qPCR analysis of $1 / 12 a$ and $I / 12 b$ expression in all sorted DC subsets before and after 48 hours of immunization; $n=3$; data represent mean \pm SEM. (C and $\mathbf{E}$ ) Unpaired, 2 -tailed Student's $t$ test; ${ }^{*} P<0.05$. (D) One-way ANOVA with multiple comparisons and Tukey's post hoc test; ${ }^{*} P<0.05$.

In this study, we found that endogenous DCs were critically required to induce polarized Th1 responses and enhance Th0 priming by vaccine DCs. We were able to identify XCR1 $1^{+}$lymph node-resident $\mathrm{CDC} 1 \mathrm{~s}$ as communication partners that take up the message delivered by the injected vaccine DC and are responsible for promoting full-blown Th1 responses. This opens up a potentially new level of complexity when considering strategies for vaccine DC optimization. The requirement of endogenous DCs for optimal antitumor DC vaccination is of clinical importance, since these patients are treated with immunosuppressive chemotherapy and are subjected to $\gamma$-irradiation that will affect endogenous DC populations. In contrast, the use of CTLA-4- and PD-1-targeted checkpoint inhibitors would not negatively affect endogenous DCs.

This study also sheds light on the question why the IL-1 $\beta / \mathrm{TNF}-\alpha / \mathrm{IL}-6 / \mathrm{PGE}_{2}$ matured vaccine DCs are successful in Th1 priming, despite a lack of IL-12-producing capacity $(29,30)$. This was also reported for BM-DC immunization of Leishmania major-challenged mice, where IL-12 production by BM-DCs was not required and, rather, recipient IL-12 was mandatory. The recipient source of IL-12 was not identified (31). The same group showed that immunization with antigen-pulsed Langerhans cells strictly required their ability to 
produce IL-12 (63), indicating that the source of IL-12 required for optimal Th1 polarization changes depending on the DC subset. Although IL-12p70 production by vaccine DCs correlates with better immune responses in tumor patients (64-66), it remained open whether this vaccine DC-derived IL-12p70 promotes adaptive T cell responses or acts locally on innate NK cells, as observed in toxoplasma infection (67). Another clinical study shows a positive correlation between IL-12p70 production by the DCs before intranodal injection and the time to progression. However, the IL-12p70 production does not correlate with the IFN- $\gamma$ ELISPOT responses of the $\mathrm{CD}^{+}$or $\mathrm{CD}^{+} \mathrm{T}$ cells of the patients (68). These findings also argue for a $\mathrm{T}$ cell-independent benefit of IL-12p70 production by the DCs for the patients. Of note, this intranodal injection does not require DC migration to the lymph nodes, and DCs may not have to decide between migration or cytokine secretion programs (32). Others found a positive correlation between IL-12p70 production by the DCs before i.v. injection and the time to progression, as well as the IFN- $\gamma / \mathrm{IL}-13$ and IFN- $\gamma / \mathrm{IL}-5$ ratios of CD8 ${ }^{+} \mathrm{T}$ cells, but not CD4 ${ }^{+} \mathrm{Th} 1$ (69). Thus, although the patients benefit from IL-12p70 production by vaccine DCs, a direct effect of vaccine-derived IL-12p70 on Th1 induction remains to be shown. Both studies $(68,69)$ used MoDCs, which are the human correlates to our murine BM-DCs (17) but differ from our setting by their intranodal or i.v. injection.

Other studies have shown IL-12-independent Th1 priming pathways, such as TNF receptor family members CD27 and OX40 on T cells interacting with their ligands CD70 and OX40L (70, 71). In our study, IL-12 production strongly supported Th1 polarization, and the $\mathrm{XCR} 1^{+}$migratory $\mathrm{cDC} 1 \mathrm{~s}$ were the mediators of this function. These cells appear to take over the Th1 polarization function from the injected BM-DCs at a later stage of the $\mathrm{T}$ cell response, when the initial antigen presentation phase is terminated and $\mathrm{T}$ cells enter their proliferative phase (42).

Interestingly, we also find that $\mathrm{CD} 103^{+} \mathrm{XCR} 1^{+}$resident $\mathrm{cDC} 1 \mathrm{~s}$ were required for antigen presentation later in the $\mathrm{T}$ cell response, indicating antigen transfer from the injected BM-DCs. This is not caused by handover of antigen to $\mathrm{CD} 103^{+} \mathrm{XCR} 1^{+}$migratory $\mathrm{CDC} 1 \mathrm{~s}$ in the skin, as observed for injected apoptotic DCs $(72,73)$, since no $\mathrm{T}$ cell priming or polarization occurred when antigen-loaded $C \mathrm{cr} 7^{-/}$BM-DC were used for immunization. Several mechanisms of antigen transfer between different DC subsets have been suggested - for example, via trogocytosis (74) or via exosomes (75) - and both pathways have been implicated in the transfer of peptide-bound MHC molecules and costimulatory molecules. Studies on viral models such as herpes simplex virus (HSV) have shown that viral antigens are handed over to XCR $1^{+}$ lymph node-resident cross-presenting DCs for an optimum CTL response $(56,76)$. The requirement for endogenous DCs to support optimal CD4 ${ }^{+} \mathrm{T}$ cell responses by DC-DC contacts in lymph nodes has been observed before, but the endogenous DC subset was not identified and further bystander function for Th1 polarization was not investigated (77). Our data suggest that migrated BM-DCs transfer antigen and Th1 polarizing information specifically to XCR $1^{+}$bystander-activated DCs in the lymph node.

In the context of $\mathrm{CpG}$ adjuvanted immunization relying exclusively on endogenous DCs, large numbers of monocytes are mobilized to lymph nodes $(78,79)$, and MoDCs were identified as the major source of IL-12, supporting $\mathrm{CD} 4^{+}$and $\mathrm{CD} 8^{+} \mathrm{T}$ cell responses (80). We show that the $\mathrm{XCR} 1^{+} \mathrm{DC}$ subset is required for Th1 polarization, which highlights the importance of identifying the roles of different DC subsets depending on the context. Here, for $\mathrm{CD} 4^{+} \mathrm{Th} 1$ polarization, it comes as no surprise that $\mathrm{XCR} 1^{+} \mathrm{CDC} 1 \mathrm{~s}$ are the subset interacting with vaccine DCs, since they were shown to be the major IL-12-producing subset under Th1 priming conditions in different tissues $(81,82)$.

Here, we evaluated how the transcriptome of steady state $\mathrm{MHC}-\mathrm{II}{ }^{\text {hi }} \mathrm{cDC} 1 \mathrm{~s}$ changes after generation of Th1 priming bystander DCs. During the steady state, MHC-II ${ }^{\text {hi }}$ DCs within lymph nodes represent tolerogenic migratory DCs (steady state migratory DCs; ssmDCs) that appear as a semimature stage with upregulated levels of nuclear RelB and surface MHC-II, CD86, CD40, and CCR7 molecules. However, all of these markers are expressed lower, as compared with pathogen- or inflammation-matured migratory DCs $(5,7,83)$. In addition, ssmDCs express a TGF- $\beta$ to induce Tregs, whereas pathogen-matured DCs produce proinflammatory cytokines to polarize CD4 ${ }^{+}$Th cell responses (33, 84). The dermal ssm-cDC1 subset, identified by expression of XCR1, CD103, and Langerin, has been characterized transcriptionally and revealed a matured phenotype with expression of RelB, IL-12p40, and CCR7 (84). Functionally, we found earlier that the ssm-cDC1s converted naive CD4 ${ }^{+} \mathrm{T}$ cells into Foxp $3^{+}$peripheral Tregs in a TGF- $\beta$-dependent manner in the skin draining lymph nodes (5). This TGF- $\beta$ signature was later confirmed at the transcriptome level (84). We found that immunogenic bystander $\mathrm{cDC} 1 \mathrm{~s}$ did not markedly upregulate typical RNA signatures or markers for DC maturation; however, the transcriptional TGF- $\beta$ signature decreased. This indicates that functionally tolerogenic 
$\mathrm{XCR} 1^{+} \mathrm{ssm}-\mathrm{cDC} 1 \mathrm{~s}$ may be reprogrammed in the lymph node to become immunogenic bystander DCs. Functional plasticity of ssm-Langerhans cells had been shown before by their continued capacity to internalize antigens after migration into the draining lymph nodes (85). Our previous data show that BM-DCs that were matured with the inflammatory stimulus TNF maintained maturation plasticity, since they could be further stimulated by LPS in vitro to release IL-12p70 or in vivo by endogenous stimuli after s.c. injection to polarize for Th1 responses (33) instead of inducing tolerance by i.v. injection (86). Together, the transcriptional changes in bystander $\mathrm{cDC} 1 \mathrm{~s}$ indicate that they downregulate tolerogenic steady-state functions and become activated to induce Th1 responses. The data provide evidence that XCR $1^{+}$lymph node-resident $\mathrm{CDC} 1 \mathrm{~s}$ can undergo functional reprogramming and activation into mature bystander DCs by LPS from the migrated BM-DCs or other bystander signals sensed in the lymph node that appeared inflamed with increased cellularity.

Our data indicate that only IL-12 production by endogenous $\mathrm{CDC} 1$ in the draining lymph nodes, but not the injected DCs, instructs Th1 polarization. The exocytosis of IL-12 is mediated by the SNARE family member VAMP7 (87). Among the upregulated genes, we identified Tmem79, which is reported to be involved in exocytosis (44). It remains to be tested whether Tmem 79 also is involved in this process.

A significant increase in $I L 12 a$ and $I L 12 b$ gene expression by quantitative PCR (qPCR) that we detected specifically in XCR $1^{+}$migratory DCs after BM-DC immunization was not observed by RNA-seq. While the low number of DCs used for sequencing can attribute to such a discrepancy, it is also possible that the bystander activation signal was diluted by the remaining steady-state XCR $1^{+}$migratory $\mathrm{cDC} 1 \mathrm{~s}$ that still carry out their tolerogenic functions. Such a heterogeneity has been observed by single cell sequencing among LPS-stimulated spleen cells, where the DCs clustered differently when compared with the existing marker-based classification (88). Using our defined transcriptional signature for bystander-activated XCR $1^{+}$ migratory $\mathrm{cDC} 1 \mathrm{~s}$, it might be possible to distinguish them from their steady-state counterpart and specifically target them for enhancing DC vaccination protocols.

In conclusion, our data suggest that Th0 priming strictly requires the vaccine DCs but endogenous bystander DCs for IL-12p70 production and Th1 polarization in the OVA/OT-II model (Supplemental Figure 4). Only the CCR7- resident, and not the migratory fraction, of the $\mathrm{XCR} 1^{+} \mathrm{CDC} 1$ subset acquired bystander function. Although DC phenotypes and functions in the lymph nodes may change in mice with established tumors or ongoing infections, these findings may be of translational importance but require confirmation in mouse tumor models and human DC vaccination studies in immunocompromised tumor patients.

\section{Methods}

Supplemental Methods are available online with this article.

\section{Study approval}

All animal experiments were performed according to the German animal protection law, as well as after approval and under control of the local authorities.

\section{Author contributions}

MBL and FE designed the experiments; DA, PA, and VP conducted experiments and acquired data; DA, $\mathrm{PA}, \mathrm{KUF}$, and FE analyzed data; $\mathrm{AB}$ and TK provided reagents; and DA and MBL wrote the manuscript.

\section{Acknowledgments}

This project was supported by the Wilhelm Sander-Foundation, the Graduate School of Life Sciences (GSLS), University of Würzburg, the DAAD STIBET program, and the Interdisciplinary Center for Clinical Research (IZKF) at the University of Würzburg (Z-6). This publication was cofunded by the University of Würzburg in the funding program Open Access Publishing. We thank Gottfried Alber and Wolfgang Kastenmüller for providing mice and Wolfgang Kastenmüller also for critical reading of the manuscript and valuable suggestions. We are also grateful to Nora Müller for her help with the confocal microscope and Sarah Eickhoff for help with the Imaris analyses.

Address correspondence to: Manfred B. Lutz, Institute for Virology and Immunobiology, Versbacherstrasse 7, 97080 Würzburg, Germany. Phone: 49.931.31.81553; Email: m.lutz@vim.uni-wuerzburg.de. 
1. Banchereau J, Steinman RM. Dendritic cells and the control of immunity. Nature. 1998;392(6673):245-252.

2. Merad M, Sathe P, Helft J, Miller J, Mortha A. The dendritic cell lineage: ontogeny and function of dendritic cells and their subsets in the steady state and the inflamed setting. Annu Rev Immunol. 2013;31:563-604.

3. Guilliams M, et al. Unsupervised High-Dimensional Analysis Aligns Dendritic Cells across Tissues and Species. Immunity. 2016;45(3):669-684.

4. Dudziak D, et al. Differential antigen processing by dendritic cell subsets in vivo. Science. 2007;315(5808):107-111.

5. Azukizawa H, et al. Steady state migratory RelB+ langerin + dermal dendritic cells mediate peripheral induction of antigen-specific CD4+ CD25+ Foxp3+ regulatory T cells. Eur J Immunol. 2011;41(5):1420-1434.

6. Pletinckx K, Döhler A, Pavlovic V, Lutz MB. Role of dendritic cell maturity/costimulation for generation, homeostasis, and suppressive activity of regulatory T cells. Front Immunol. 2011;2:39.

7. Idoyaga J, et al. Specialized role of migratory dendritic cells in peripheral tolerance induction. J Clin Invest. 2013;123(2):844-854.

8. Döhler A, et al. RelB ${ }^{+}$Steady-State Migratory Dendritic Cells Control the Peripheral Pool of the Natural Foxp $3^{+}$Regulatory T Cells. Front Immunol. 2017;8:726.

9. Zhu J, Yamane H, Paul WE. Differentiation of effector CD4 T cell populations ( $\left.{ }^{*}\right)$. Annu Rev Immunol. 2010;28:445-489.

10. Durai V, Murphy KM. Functions of Murine Dendritic Cells. Immunity. 2016;45(4):719-736.

11. Swiecki M, Gilfillan S, Vermi W, Wang Y, Colonna M. Plasmacytoid dendritic cell ablation impacts early interferon responses and antiviral NK and CD8(+) T cell accrual. Immunity. 2010;33(6):955-966.

12. Tussiwand R, et al. Klf4 expression in conventional dendritic cells is required for T helper 2 cell responses. Immunity. 2015;42(5):916-928.

13. Lewis KL, et al. Notch2 receptor signaling controls functional differentiation of dendritic cells in the spleen and intestine. Immunity. 2011;35(5):780-791.

14. Waithman J, et al. Resident CD8(+) and migratory CD103(+) dendritic cells control CD8 T cell immunity during acute influenza infection. PLoS One. 2013;8(6):e66136.

15. Plantinga $\mathrm{M}$, et al. Conventional and monocyte-derived $\mathrm{CD} 1 \mathrm{~b}(+)$ dendritic cells initiate and maintain $\mathrm{T}$ helper 2 cell-mediated immunity to house dust mite allergen. Immunity. 2013;38(2):322-335.

16. Cheong C, et al. Microbial stimulation fully differentiates monocytes to DC-SIGN/CD209(+) dendritic cells for immune T cell areas. Cell. 2010;143(3):416-429.

17. Lutz MB, Strobl H, Schuler G, Romani N. GM-CSF Monocyte-Derived Cells and Langerhans Cells As Part of the Dendritic Cell Family. Front Immunol. 2017;8:1388.

18. Pletinckx K, et al. Similar inflammatory DC maturation signatures induced by TNF or Trypanosoma brucei antigens instruct default Th2-cell responses. Eur J Immunol. 2011;41(12):3479-3494.

19. Silva-Vilches C, et al. Low doses of cholera toxin and its mediator cAMP induce CTLA-2 secretion by dendritic cells to enhance regulatory T cell conversion. PLoS One. 2017;12(7):e0178114.

20. Openshaw $\mathrm{P}$, et al. Heterogeneity of intracellular cytokine synthesis at the single-cell level in polarized T helper 1 and $\mathrm{T}$ helper 2 populations. J Exp Med. 1995;182(5):1357-1367.

21. Kaliński P, Hilkens CM, Wierenga EA, Kapsenberg ML. T-cell priming by type-1 and type-2 polarized dendritic cells: the concept of a third signal. Immunol Today. 1999;20(12):561-567.

22. Macatonia SE, et al. Dendritic cells produce IL-12 and direct the development of Th1 cells from naive CD4+ T cells. J Immunol. 1995;154(10):5071-5079.

23. Reis e Sousa C, et al. In vivo microbial stimulation induces rapid CD40 ligand-independent production of interleukin 12 by dendritic cells and their redistribution to T cell areas. J Exp Med. 1997;186(11):1819-1829.

24. Ho CS, Munster D, Pyke CM, Hart DN, López JA. Spontaneous generation and survival of blood dendritic cells in mononuclear cell culture without exogenous cytokines. Blood. 2002;99(8):2897-2904.

25. Nestle FO, et al. Vaccination of melanoma patients with peptide- or tumor lysate-pulsed dendritic cells. Nat Med. 1998;4(3):328-332.

26. Alberts-Grill N, et al. Dendritic Cell KLF2 Expression Regulates T Cell Activation and Proatherogenic Immune Responses. J Immunol. 2016;197(12):4651-4662.

27. Gross S, et al. Twelve-year survival and immune correlates in dendritic cell-vaccinated melanoma patients. JCI Insight. 2017;2(8):91438.

28. Jonuleit $\mathrm{H}$, et al. Pro-inflammatory cytokines and prostaglandins induce maturation of potent immunostimulatory dendritic cells under fetal calf serum-free conditions. Eur J Immunol. 1997;27(12):3135-3142.

29. Lee AW, et al. A clinical grade cocktail of cytokines and PGE2 results in uniform maturation of human monocyte-derived dendritic cells: implications for immunotherapy. Vaccine. 2002;20 Supp1 4:A8-A22.

30. Schuler-Thurner B, et al. Rapid induction of tumor-specific type $1 \mathrm{~T}$ helper cells in metastatic melanoma patients by vaccination with mature, cryopreserved, peptide-loaded monocyte-derived dendritic cells. J Exp Med. 2002;195(10):1279-1288.

31. Ramírez-Pineda JR, Fröhlich A, Berberich C, Moll H. Dendritic cells (DC) activated by CpG DNA ex vivo are potent inducers of host resistance to an intracellular pathogen that is independent of IL-12 derived from the immunizing DC. J Immunol. 2004;172(10):6281-6289.

32. Luft $\mathrm{T}$, et al. Tuning the volume of the immune response: strength and persistence of stimulation determine migration and cytokine secretion of dendritic cells. Blood. 2004;104(4):1066-1074.

33. Voigtländer C, et al. Dendritic cells matured with TNF can be further activated in vitro and after subcutaneous injection in vivo which converts their tolerogenicity into immunogenicity. J Immunother. 2006;29(4):407-415.

34. Brewitz A, et al. CD8 ${ }^{+}$T Cells Orchestrate pDC-XCR $1^{+}$Dendritic Cell Spatial and Functional Cooperativity to Optimize Priming. Immunity. 2017;46(2):205-219.

35. Baumjohann D, Hess A, Budinsky L, Brune K, Schuler G, Lutz MB. In vivo magnetic resonance imaging of dendritic cell migration into the draining lymph nodes of mice. Eur J Immunol. 2006;36(9):2544-2555.

36. Reinhardt RL, Hong S, Kang SJ, Wang ZE, Locksley RM. Visualization of IL-12/23p40 in vivo reveals immunostimulatory dendritic cell migrants that promote Th1 differentiation. J Immunol. 2006;177(3):1618-1627.

37. Guilliams M, et al. Skin-draining lymph nodes contain dermis-derived CD103(-) dendritic cells that constitutively produce retinoic 
acid and induce Foxp3(+) regulatory T cells. Blood. 2010;115(10):1958-1968.

38. Gillessen S, et al. Mouse interleukin-12 (IL-12) p40 homodimer: a potent IL-12 antagonist. Eur J Immunol. 1995;25(1):200-206.

39. Yamazaki C, et al. Critical roles of a dendritic cell subset expressing a chemokine receptor, XCR1. J Immunol. 2013;190(12):6071-6082.

40. Lahoud MH, et al. DEC-205 is a cell surface receptor for CpG oligonucleotides. Proc Natl Acad Sci USA. 2012;109(40):1627016275.

41. Lutz MB, et al. An advanced culture method for generating large quantities of highly pure dendritic cells from mouse bone marrow. J Immunol Methods. 1999;223(1):77-92.

42. Mempel TR, Henrickson SE, Von Andrian UH. T-cell priming by dendritic cells in lymph nodes occurs in three distinct phases. Nature. 2004;427(6970):154-159.

43. Miller MJ, Safrina O, Parker I, Cahalan MD. Imaging the single cell dynamics of CD4+ T cell activation by dendritic cells in lymph nodes. J Exp Med. 2004;200(7):847-856.

44. Sasaki T, et al. A homozygous nonsense mutation in the gene for Tmem79, a component for the lamellar granule secretory system produces spontaneous eczema in an experimental model of atopic dermatitis. J Allergy Clin Immunol. 2013;132(5):1111-1120.e4.

45. Redmann V, et al. Clec16a is Critical for Autolysosome Function and Purkinje Cell Survival. Sci Rep. 2016;6:23326.

46. van Luijn MM, et al. Multiple sclerosis-associated CLEC16A controls HLA class II expression via late endosome biogenesis. Brain. 2015;138(Pt 6):1531-1547.

47. Landuyt AE, Klocke BJ, Colvin TB, Schoeb TR, Maynard CL. Cutting Edge: ICOS-Deficient Regulatory T Cells Display Normal Induction of Il10 but Readily Downregulate Expression of Foxp3. J Immunol. 2019;202(4):1039-1044.

48. Widjaja-Adhi MAK, et al. Transcription factor ISX mediates the cross talk between diet and immunity. Proc Natl Acad Sci USA 2017;114(43):11530-11535.

49. Divangahi $\mathrm{M}$, et al. Critical negative regulation of type $1 \mathrm{~T}$ cell immunity and immunopathology by signaling adaptor DAP12 during intracellular infection. J Immunol. 2007;179(6):4015-4026.

50. Lanier LL. DAP10- and DAP12-associated receptors in innate immunity. Immunol Rev. 2009;227(1):150-160.

51. Sumpter TL, Packiam V, Turnquist HR, Castellaneta A, Yoshida O, Thomson AW. DAP12 promotes IRAK-M expression and IL-10 production by liver myeloid dendritic cells and restrains their T cell allostimulatory ability. J Immunol. 2011;186(4):1970-1980

52. Nieves W, et al. Myeloid-Restricted AMPK $\alpha 1$ Promotes Host Immunity and Protects against IL-12/23p40-Dependent Lung Injury during Hookworm Infection. J Immunol. 2016;196(11):4632-4640.

53. Kouzaki H, Ijima K, Kobayashi T, O'Grady SM, Kita H. The danger signal, extracellular ATP, is a sensor for an airborne allergen and triggers IL-33 release and innate Th2-type responses. J Immunol. 2011;186(7):4375-4387.

54. Nakayama M. Antigen Presentation by MHC-Dressed Cells. Front Immunol. 2014;5:672.

55. Muzaki AR, et al. Intestinal CD103(+)CD11b(-) dendritic cells restrain colitis via IFN- $\gamma$-induced anti-inflammatory response in epithelial cells. Mucosal Immunol. 2016;9(2):336-351.

56. Allan RS, et al. Migratory dendritic cells transfer antigen to a lymph node-resident dendritic cell population for efficient CTL priming. Immunity. 2006;25(1):153-162.

57. Yewdall AW, Drutman SB, Jinwala F, Bahjat KS, Bhardwaj N. CD8+ T cell priming by dendritic cell vaccines requires antigen transfer to endogenous antigen presenting cells. PLOS ONE. 2010;5(6):e11144.

58. Morse MA, et al. Phase I study of immunization with dendritic cells modified with fowlpox encoding carcinoembryonic antigen and costimulatory molecules. Clin Cancer Res. 2005;11(8):3017-3024.

59. Hodge JW, Grosenbach DW, Aarts WM, Poole DJ, Schlom J. Vaccine therapy of established tumors in the absence of autoimmunity. Clin Cancer Res. 2003;9(5):1837-1849.

60. Okada N, et al. Immunological properties and vaccine efficacy of murine dendritic cells simultaneously expressing melanoma-associated antigen and interleukin-12. Cancer Gene Ther. 2005;12(1):72-83.

61. Turnis ME, et al. IRAK-M removal counteracts dendritic cell vaccine deficits in migration and longevity. J Immunol. 2010;185(7):4223-4232.

62. Soares KC, et al. PD-1/PD-L1 blockade together with vaccine therapy facilitates effector T-cell infiltration into pancreatic tumors. J Immunother. 2015;38(1):1-11

63. Berberich C, Ramírez-Pineda JR, Hambrecht C, Alber G, Skeiky YA, Moll H. Dendritic cell (DC)-based protection against an intracellular pathogen is dependent upon DC-derived IL-12 and can be induced by molecularly defined antigens. J Immunol. 2003; 170(6):3171-3179

64. Wilgenhof S, et al. Therapeutic vaccination with an autologous mRNA electroporated dendritic cell vaccine in patients with advanced melanoma. J Immunother. 2011;34(5):448-456.

65. Linette GP, Carreno BM. Dendritic cell-based vaccines: Shining the spotlight on signal 3. Oncoimmunology. 2013;2(11):e26512.

66. Castiello L, et al. Expression of CD14, IL10, and Tolerogenic Signature in Dendritic Cells Inversely Correlate with Clinical and Immunologic Response to TARP Vaccination in Prostate Cancer Patients. Clin Cancer Res. 2017;23(13):3352-3364.

67. Ivanova DL, Mundhenke TM, Gigley JP. The IL-12- and IL-23-Dependent NK Cell Response Is Essential for Protective Immunity against Secondary Toxoplasma gondii Infection. J Immunol. 2019;203(11):2944-2958.

68. Okada H, et al. Induction of CD8+ T-cell responses against novel glioma-associated antigen peptides and clinical activity by vaccinations with \{alpha\}-type 1 polarized dendritic cells and polyinosinic-polycytidylic acid stabilized by lysine and carboxymethylcellulose in patients with recurrent malignant glioma. J Clin Oncol. 2011;29(3):330-336.

69. Carreno BM, et al. IL-12p70-producing patient DC vaccine elicits Tc1-polarized immunity. J Clin Invest. 2013;123(8):3383-3394

70. Sanchez PJ, Kedl RM. An alternative signal 3: CD8 ${ }^{+} \mathrm{T}$ cell memory independent of IL-12 and type I IFN is dependent on CD27/OX40 signaling. Vaccine. 2012;30(6):1154-1161

71. Soares H, et al. A subset of dendritic cells induces CD4+ T cells to produce IFN-gamma by an IL-12-independent but CD70-dependent mechanism in vivo. $J$ Exp Med. 2007;204(5):1095-1106.

72. Desch AN, et al. CD103+ pulmonary dendritic cells preferentially acquire and present apoptotic cell-associated antigen. $J$ Exp Med. 2011;208(9):1789-1797.

73. Inaba K, et al. Efficient presentation of phagocytosed cellular fragments on the major histocompatibility complex class II products 
of dendritic cells. J Exp Med. 1998;188(11):2163-2173.

74. Zhang QJ, et al. Trogocytosis of MHC-I/peptide complexes derived from tumors and infected cells enhances dendritic cell cross-priming and promotes adaptive T cell responses. PLoS One. 2008;3(8):e3097.

75. Segura E, Guérin C, Hogg N, Amigorena S, Théry C. CD8+ dendritic cells use LFA-1 to capture MHC-peptide complexes from exosomes in vivo. J Immunol. 2007;179(3):1489-1496.

76. Eickhoff S, et al. Robust Anti-viral Immunity Requires Multiple Distinct T Cell-Dendritic Cell Interactions. Cell. 2015;162(6):1322-1337.

77. Kleindienst P, Brocker T. Endogenous dendritic cells are required for amplification of $\mathrm{T}$ cell responses induced by dendritic cell vaccines in vivo. J Immunol. 2003;170(6):2817-2823.

78. Chakarov S, Fazilleau N. Monocyte-derived dendritic cells promote T follicular helper cell differentiation. EMBO Mol Med. 2014;6(5):590-603.

79. Kwissa M, Nakaya HI, Oluoch H, Pulendran B. Distinct TLR adjuvants differentially stimulate systemic and local innate immune responses in nonhuman primates. Blood. 2012;119(9):2044-2055.

80. De Koker S, et al. Inflammatory monocytes regulate Th1 oriented immunity to CpG adjuvanted protein vaccines through production of IL-12. Sci Rep. 2017;7(1):5986.

81. Conejero L, Khouili SC, Martínez-Cano S, Izquierdo HM, Brandi P, Sancho D. Lung CD103+ dendritic cells restrain allergic airway inflammation through IL-12 production. JCI Insight. 2017;2(10):90420.

82. Moretto MM, Harrow DI, Hawley TS, Khan IA. Interleukin-12-producing CD103+ CD11b- CD8+ dendritic cells are responsible for eliciting gut intraepithelial lymphocyte response against Encephalitozoon cuniculi. Infect Immun. 2015;83(12):4719-4730

83. Ohl L, et al. CCR7 governs skin dendritic cell migration under inflammatory and steady-state conditions. Immunity 2004;21(2):279-288

84. Ardouin L, et al. Broad and Largely Concordant Molecular Changes Characterize Tolerogenic and Immunogenic Dendritic Cell Maturation in Thymus and Periphery. Immunity. 2016;45(2):305-318.

85. Ruedl C, Koebel P, Karjalainen K. In vivo-matured Langerhans cells continue to take up and process native proteins unlike in vitro-matured counterparts. J Immunol. 2001;166(12):7178-7182.

86. Menges $\mathrm{M}$, et al. Repetitive injections of dendritic cells matured with tumor necrosis factor alpha induce antigen-specific protec tion of mice from autoimmunity. J Exp Med. 2002;195(1):15-21.

87. Chiaruttini G, et al. The SNARE VAMP7 Regulates Exocytic Trafficking of Interleukin-12 in Dendritic Cells. Cell Rep. 2016;14(11):2624-2636

88. Jaitin DA, et al. Massively parallel single-cell RNA-seq for marker-free decomposition of tissues into cell types. Science. 2014;343(6172):776-779.

89. Nguyen NT, et al. Aryl hydrocarbon receptor negatively regulates dendritic cell immunogenicity via a kynurenine-dependent mechanism. Proc Natl Acad Sci USA. 2010;107(46):19961-19966.

90. Whitmore MM, Iparraguirre A, Kubelka L, Weninger W, Hai T, Williams BR. Negative regulation of TLR-signaling pathways by activating transcription factor-3. J Immunol. 2007;179(6):3622-3630.

91. Ebner S, et al. A novel role for IL-3: human monocytes cultured in the presence of IL-3 and IL-4 differentiate into dendritic cells that produce less IL-12 and shift Th cell responses toward a Th2 cytokine pattern. J Immunol. 2002;168(12):6199-6207.

92. Guimond $\mathrm{M}$, et al. Interleukin 7 signaling in dendritic cells regulates the homeostatic proliferation and niche size of CD4+ T cells. Nat Immunol. 2009;10(2):149-157.

93. Amsen D, Blander JM, Lee GR, Tanigaki K, Honjo T, Flavell RA. Instruction of distinct CD4 T helper cell fates by different notch ligands on antigen-presenting cells. Cell. 2004;117(4):515-526.

94. Endo S, Sakamoto Y, Kobayashi E, Nakamura A, Takai T. Regulation of cytotoxic T lymphocyte triggering by PIR-B on dendritic cells. Proc Natl Acad Sci USA. 2008;105(38):14515-14520.

95. Bell BD, et al. The transcription factor STAT5 is critical in dendritic cells for the development of TH2 but not TH1 responses. Nat Immunol. 2013;14(4):364-371.

96. Arima K, Watanabe N, Hanabuchi S, Chang M, Sun SC, Liu YJ. Distinct signal codes generate dendritic cell functional plasticity. Sci Signal. 2010;3(105):ra4.

97. Ramalingam R, et al. Dendritic cell-specific disruption of TGF- $\beta$ receptor II leads to altered regulatory T cell phenotype and spontaneous multiorgan autoimmunity. J Immunol. 2012;189(8):3878-3893.

98. Kabashima K, Sugita K, Shiraishi N, Tamamura H, Fujii N, Tokura Y. CXCR4 engagement promotes dendritic cell survival and maturation. Biochem Biophys Res Commun. 2007;361(4):1012-1016.

99. Maddaluno L, et al. The adhesion molecule L1 regulates transendothelial migration and trafficking of dendritic cells. $J$ Exp Med. 2009;206(3):623-635.

100. Azukizawa H, et al. Steady state migratory RelB+ langerin+ dermal dendritic cells mediate peripheral induction of antigen-specific CD4+ CD25+ Foxp3+ regulatory T cells. Eur J Immunol. 2011;41(5):1420-1434.

101. Park HE, Shin MK, Park HT, Jung M, Cho YI, Yoo HS. Gene expression profiles of putative biomarker candidates in Mycobacterium avium subsp. paratuberculosis-infected cattle. Pathog Dis. 2016;74(4):ftw022.

102. Kim SJ, et al. Increased cathepsin S in Prdm $1 \%$ dendritic cells alters the $\mathrm{T}_{\mathrm{FH}}$ cell repertoire and contributes to lupus. Nat Immunol. 2017;18(9):1016-1024.

103. Vremec D, Shortman K. What's in a Name? Some Early and Current Issues in Dendritic Cell Nomenclature. Front Immunol. $2015 ; 6: 267$

104. Wang C, Kang SG, HogenEsch H, Love PE, Kim CH. Retinoic acid determines the precise tissue tropism of inflammatory Th17 cells in the intestine. J Immunol. 2010;184(10):5519-5526.

105. Satpathy AT, et al. Runx1 and Cbf $\beta$ regulate the development of Flt3+ dendritic cell progenitors and restrict myeloproliferative disorder. Blood. 2014;123(19):2968-2977.

106. Sawai CM, et al. Transcription factor Runx2 controls the development and migration of plasmacytoid dendritic cells. $J$ Exp Med. 2013;210(11):2151-2159. 